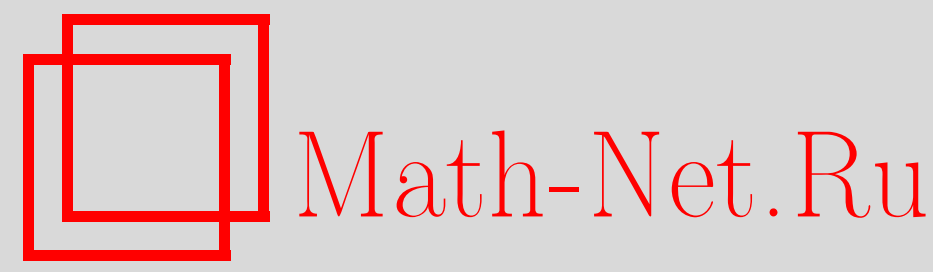

К. П. Исаев, Р. С. Юлмухаметов, Преобразования Лапласа функционалов на пространствах Бергмана, Изв. РАН. Сер. матем., 2004, том 68, выпуск 1, 5-42

DOI: https://doi.org/10.4213/im465

Использование Общероссийского математического портала Math-Net.Ru подразумевает, что вы прочитали и согласны с пользовательским соглашением

http://www . mathnet.ru/rus/agreement

Параметры загрузки:

IP : 3.95 .254 .165

26 апреля 2023 г., 12:10:34 
УДК 517.54

\author{
К.П. Исаев, Р.С. Юлмухаметов
}

\title{
Преобразования Лапласа функционалов на пространствах Бергмана
}

\footnotetext{
Приведено полное описание пространства, сопряженного к пространству Бергмана на плоской ограниченной выпуклой области.

Библиография: 17 наименований.
}

\section{Введение}

Пусть $D$ - ограниченная выпуклая область на комплексной плоскости. Через $B_{2}(D)$ обозначим пространство Бергмана, состоящее из функций, голоморфных в $D$ и интегрируемых с квадратом по плошади $D$. Пространство $B_{2}(D)$ является гильбертовым со скалярным произведением

$$
(f, g)=\int_{D} f(z) \overline{g(z)} d v(z)
$$

где $d v$ - элемент площади. Функция $e^{\lambda z}$ при любом $\lambda \in \mathbb{C}$ принадлежит $B_{2}(D)$, и система $\left\{e^{\lambda z}, \lambda \in \mathbb{C}\right\}$ полна в $B_{2}(D)[1]$. Если $S$ - линейный непрерывный функционал на $B_{2}(D)$, то функция

$$
\widehat{S}(\lambda)=S\left(e^{\lambda z}\right), \quad \lambda \in \mathbb{C},
$$

называется преобразованием Лапласа функционала $S$. Из легко проверяемой оценки

$$
\max _{z \in D}\left|\frac{e^{\lambda z}-e^{\lambda_{0} z}}{\lambda-\lambda_{0}}-z e^{\lambda_{0} z}\right| \leqslant\left|\lambda-\lambda_{0}\right| \max _{z \in D}\left|z^{2} e^{\lambda z}\right|
$$

следует, что при $\lambda \rightarrow \lambda_{0}$

$$
\left\|\frac{e^{\lambda z}-e^{\lambda_{0} z}}{\lambda-\lambda_{0}}-z e^{\lambda_{0} z}\right\|_{B_{2}(D)} \rightarrow 0
$$

Значит, функция $\widehat{S}(\lambda)$ является целой, причем $\widehat{S}^{\prime}(\lambda)=S\left(z e^{\lambda z}\right)$.

Определим отображение $L: B_{2}^{*}(D) \rightarrow H(\mathbb{C})$, сопоставляя каждому функционалу $S \in B_{2}^{*}(D)$ его преобразование Лапласа $\widehat{S}(\lambda)$. Полнота системы $\left\{e^{\lambda z}, \lambda \in \mathbb{C}\right\}$ означает, что отображение $L$ действует инъективно.

Цель настоящей работы - определить образ отображения $L$. 
Аналогичная задача была выполнена в работе [2], в которой рассматривалось пространство Смирнова $E_{2}(D)$, определяемое как пополнение пространства полиномов относительно нормы

$$
\|f\|^{2}=\int_{\partial D}|f(z)|^{2} d s(z)
$$

где $d s$ - элемент длины гранищы.

Задачи описания образа в более слабых топологиях рассмотрены в работах [3]-[6], для пространств Бергмана - в [7]-[10]. Задача описания образов при преобразованиях Коши и Лапласа функционалов на пространствах с более слабыми топологиями изучалась в работах [11]-[15].

Общая идея решения поставленной задачи состоит в следующем.

$\operatorname{KaK~известно,~система~}\left\{\frac{1}{z-\zeta}, \zeta \notin \bar{D}\right\}$ также полна в пространстве $B_{2}(D)[1]$. Преобразованием Кочи функиионала $S \in B_{2}^{*}(D)$ назовем функцию

$$
\widetilde{S}(\zeta)=S\left(\frac{1}{z-\zeta}\right), \quad \zeta \in \mathbb{C} \backslash \bar{D}
$$

Отображение $C: S \rightarrow \widetilde{S}$ инъективно действует из $B_{2}^{*}(D)$ в пространство

$$
H_{0}(\mathbb{C} \backslash \bar{D})=\{\gamma(\zeta) \in H(\mathbb{C} \backslash \bar{D}), \gamma(\infty)=0\}
$$

Задача определения образа отображения $C$ рассмотрена и полностью решена в работах [7]-[9]. Решение задачи описьвается следуюшей теоремой.

Теорема А. Отображсние $C$ устанавливает линейный топологический изоморфизм пространства $B_{2}^{*}(D)$ и пространства

$$
B_{2}^{1}(\mathbb{C} \backslash \bar{D})=\left\{\gamma \in H_{0}(\mathbb{C} \backslash \bar{D}): \int_{\mathbb{C} \backslash \bar{D}}\left|\gamma^{\prime}(\zeta)\right|^{2} d v(\zeta)<\infty\right\}
$$

Для функции $\gamma \in H_{0}(\mathbb{C} \backslash \bar{D})$ ее преобразованием Бореля называется целая функция

$$
B(\gamma)(\lambda)=\frac{1}{2 \pi i} \int_{\Gamma} \gamma(\zeta) e^{\lambda \zeta} d \zeta
$$

где $\Gamma$ - произвольный контур, охватываюший $D$. Имеется обратная формула для преобразования Бореля.

Пусть $h(\varphi)$ - опорная функция области $D$, т. е.

$$
h(\varphi)=\max _{z \in \bar{D}} \operatorname{Re} z e^{i \varphi}, \quad \varphi \in[0,2 \pi]
$$

Если функцию $B(\gamma)$ обозначим через $f$, то на полуплоскости $P_{\varphi}=\left\{\zeta: \operatorname{Re} \zeta e^{i \varphi}>\right.$ $h(\varphi)\}$ исходная функция $\gamma$ восстанавливается по формуле

$$
\gamma(\zeta)=\int_{0}^{\infty} f\left(r e^{i \varphi}\right) e^{-\zeta r e^{i \varphi}} e^{i \varphi} d r
$$


Преобразования Лапласа, Бореля и Коши связаны простой формулой. Действительно, возьмем композицию преобразований Бореля и Коши:

$$
\int_{\Gamma} S_{z}\left(\frac{1}{z-\zeta}\right) e^{\lambda \zeta} d \zeta=S\left(\int_{\Gamma} \frac{e^{\lambda \zeta}}{z-\zeta} d \zeta\right)=-S\left(e^{\lambda z}\right)
$$

т. е. $L=-B \circ C$. Учитывая теорему $\mathrm{A}$, мы приходим к следующей постановке вопроса: какими свойствами обладает преобразование Бореля функций из пространства $B_{2}^{1}(\mathbb{C} \backslash \bar{D})$ ?

На основе анализа результатов работы [2] мы сформулировали следующую гипотезу.

Пусть

$$
\begin{gathered}
\Delta(\varphi)=h(\varphi)+\int_{0}^{\varphi} h(\theta) d \theta, \\
K(\lambda)=\left\|e^{\lambda z}\right\|_{B_{2}(D)}^{2}=\int_{D}\left|e^{\lambda z}\right|^{2} d v(z) .
\end{gathered}
$$

Определим следуюшую норму для целых функций:

$$
\|F\|=\left(\int_{0}^{2 \pi} \int_{0}^{\infty} \frac{\left|F\left(r e^{i \varphi}\right)\right|^{2}}{K\left(r e^{i \varphi}\right)} d r d \Delta(\varphi)\right)^{1 / 2}
$$

Если $\gamma \in B_{2}^{1}(\mathbb{C} \backslash \bar{D})$ и $F$ - ее преобразование Бореля, то

$$
c\|\gamma\|_{B_{2}^{1}(\mathbb{C} \backslash \bar{D})} \leqslant\|F\| \leqslant C\|\gamma\|_{B_{2}^{1}(\mathbb{C} \backslash \bar{D})},
$$

где постоянные $c, C$ не зависят от функции $\gamma$. Эта гипотеза оказалась справедливой.

Для точной формулировки утверждения, на котором, по сушеству, построено доказательство справедливости нашей гипотезы, введем обозначения для некоторых характеристик выпуклых областей. Через $|D|$ обозначим плошадь области $D$, а через $l(D)$ - длину ее границы. Рассмотрим всевозможные прямолинейные полосы, содержащие область $D$, и через $\sigma(D)$ обозначим наименьшую ширину таких полос. Наконец, положим

$$
R(D)=\max _{z \in \bar{D}}|z| .
$$

Для функций $\gamma \in H_{0}(\mathbb{C} \backslash \bar{D})$ рассмотрим еще следуюшую норму:

$$
\|\gamma\|=\left(\int_{\mathbb{C} \backslash \bar{D}}\left|\gamma^{\prime \prime}(\zeta)\right|^{2} \operatorname{dist}^{2}(\zeta, D) d v(\zeta)\right)^{1 / 2}
$$

где $\operatorname{dist}(\zeta, D)$ - расстояние от точки $\zeta$ до границы $D$. 
ПОДГОТОВИТЕЛЬНАЯ ТЕОРЕМА. Пусть $D$ - ограниченная выпуклая область, $\gamma \in B_{2}^{1}(\mathbb{C} \backslash \bar{D})$ и $F-$ преобразование Бореля функиии $\gamma$. Тогда имеет место соотношение

$$
c(D)\|\gamma\| \leqslant\|F\| \leqslant C(D)\|\gamma\|
$$

$2 \partial e$

$$
\begin{aligned}
c(D)= & \frac{2^{-14} \sigma^{2}(D)}{A \operatorname{diam}^{2}(D)}\left(\frac{16^{4}(l(D)+\pi \sigma(D) / 2)^{2} R^{2}(D)}{\pi^{2} \sigma^{4}(D)}+1\right)^{-1}, \\
C(D)= & \frac{2^{14} \operatorname{diam}^{2}(D) l(D) R(D)}{a \sigma^{2}(D)|D|}\left(\frac{5 \cdot 16^{4}(l(D)+\pi \sigma(D) / 2)^{2}}{\pi^{2} \sigma^{4}(D)}+1\right) \\
& +\frac{2^{17} \operatorname{diam}^{4}(D)}{a|D|^{2}},
\end{aligned}
$$

причем а, $A$ - абсолютные постоянные, т. е. не зависящие от области D.

Далее докажем (теорема 6), что норма в пространстве $B_{2}^{1}(\mathbb{C} \backslash \bar{D})$ эквивалентна норме $\|\gamma\|$. Тем самым из подготовительной теоремы и теоремы А получим следующий ответ на поставленный вопрос об образе преобразования Лапласа.

ОСНОВНАЯ ТЕОРЕМА. Преобразование Лапласа устанавливает линейный непрерывныи изоморфизм пространства $B_{2}^{*}(D)$ и пространства цельх функций F с нормой

$$
\|F\|=\left(\int_{0}^{2 \pi} \int_{0}^{\infty} \frac{\left|F\left(r e^{i \varphi}\right)\right|^{2}}{K\left(r e^{i \varphi}\right)} d r d \Delta(\varphi)\right)^{1 / 2}
$$

Доказательство подготовительной теоремы разобьем на две части. В $\S 1$ при фиксированном $\varphi$ докажем равномерную по $\varphi$ эквивалентность интеграла

$$
I_{\varphi}(F)=\int_{0}^{\infty} \frac{\left|F\left(r e^{i \varphi}\right)\right|^{2}}{K\left(r e^{i \varphi}\right)} d r
$$

некоторому интегралу от $\gamma$ по полуплоскости $P_{\varphi}$. В $\S 2$ полученные оценки проинтегрируем по $\varphi$.

ЗАмЕчАнИЕ. Подготовительную теорему достаточно доказать для областей, не содержащих на своей границе прямолинейных отрезков и углов. Это следует из того, что постоянные $c(D), C(D)$ непрерьвно зависят от $D$. Действительно, допустим, что теорема доказана с указанными дополнительными условиями на область.

Пусть имеются область $D$, функция $\gamma \in B_{2}^{1}(\mathbb{C} \backslash \bar{D})$ и ее преобразование Бореля $F$. Выберем произвольное $\varepsilon>0$. Для двух плоских множеств $A, B$ через $A+B$ будем обозначать их обычную арифметическую сумму:

$$
A+B=\{z+w: z \in A, w \in B\}
$$

и пусть $B(z, r)$ - круг с центром в точке $z$ радиуса $r$. Через $D_{\varepsilon}$ обозначим сумму $D+B(0, \varepsilon)$. Впишем во множество $D_{\varepsilon}$ выпукльй многоугольник, содержащий $D$. 
Затем заменим каждую сторону этого многоугольника на дугу достаточно большой окружности так, чтобы полученная область $D^{\prime}$ оставалась выпуклой и содержалась в области $D_{2 \varepsilon}$. На границе $D^{\prime}$ нет прямолинейных участков, но есть углы. Чтобы от них избавиться, перейдем к области $D^{\prime}+B(0, \varepsilon)$. Таким образом, получим область без углов и отрезков на границе, содержащую $D$ и содержащуюся в $D_{3 \varepsilon}$.

Полагая $\varepsilon=\frac{1}{n}, n=1,2,3, \ldots$, построим указанным образом области $D_{n}$ без углов и отрезков на границе. Поскольку $D_{n} \supset D$, то $\gamma \in H_{0}\left(\mathbb{C} \backslash D_{n}\right)$ для любого $n$. Можно применить теорему, в результате получим

$$
c\left(D_{n}\right)\|\gamma\|_{n} \leqslant\|F\|_{n} \leqslant C\left(D_{n}\right)\|\gamma\|_{n},
$$

где $\|\cdot\|_{n}$ - норма, построенная по области $D_{n}$. Нетрудно убедиться в том, что при $n \rightarrow \infty$ имеем $\|\cdot\|_{n} \rightarrow\|\cdot\|$ как для функции $\gamma$, так и для функции $F$. (Для функции $\gamma$ это следует прямо из теоремы Лебега, для функции $F$ - из, например, теоремы Фату.) Остается перейти к пределу в соотношении $(*)$ и получить подготовительную теорему для области $D$.

Условия, налагаемые на границу области, потребуются лишь для более четкого изложения доказательства теоремы 2.

\section{§1. Оценка интегралов по радиусу}

Зафиксируем $\varphi \in[0,2 \pi]$. Пусть для целой функции $F(\lambda)$ конечен интеграл

$$
I_{\varphi}=\int_{0}^{\infty} \frac{\left|F\left(r e^{i \varphi}\right)\right|^{2}}{K\left(r e^{i \varphi}\right)} d r
$$

Если $F=B(\gamma)$, то $B\left(\gamma^{\prime \prime}\right)=F(\lambda) \lambda^{2}$. По обратной формуле Бореля (1) в полуплоскости $P_{\varphi}$ имеем представление

$$
\gamma^{\prime \prime}(\zeta)=\int_{0}^{\infty} F\left(r e^{i \varphi}\right) r^{2} e^{3 i \varphi} e^{-\zeta r e^{i \varphi}} d r
$$

Точки полуплоскости $P_{\varphi}$ представим в виде $\zeta=(h(\varphi)-\xi) e^{-i \varphi}$, где $\xi$ изменяется в левой полуплоскости $\operatorname{Re} \xi<0$. Тогда

$$
\gamma^{\prime \prime}\left(e^{-i \varphi}(h(\varphi)-\xi)\right)=\int_{0}^{\infty}\left[F\left(r e^{i \varphi}\right) r^{2} e^{3 i \varphi} e^{-h(\varphi) r}\right] e^{\xi r} d r .
$$

Интеграл в правой части - это преобразование Фурье-Лапласа функции $F\left(r e^{i \varphi}\right) \times$ $r^{2} e^{3 i \varphi} e^{-h(\varphi) r}$. Для оценки $\gamma^{\prime \prime}$ мы намерены применить следующую теорему (см. [2]) о весовых преобразованиях Фурье-Лапласа.

Теорема В. Пусть и(t) - выпуклая функиия, заданная на интервале I, $\widetilde{u}(x)-$ функиия, сопряженная по Юнгу с и(t):

$$
\widetilde{u}(x)=\sup _{t \in I}(x t-u(t))
$$


Положим

$$
\begin{aligned}
J & =\{x \in \mathbb{R}: \widetilde{u}(x)<\infty\}, \\
K_{0}(x) & =\int_{I} e^{2 t x-2 u(t)} d t, \quad x \in J .
\end{aligned}
$$

Тогда для любой функиии g на I, для которой конечен интеграл

$$
\|g\|^{2}=\int_{I}|g(t)|^{2} e^{-2 u(t)} d t,
$$

функция $\widehat{g}(z)=\int_{I} \overline{g(t)} e^{z t-2 u(t)} d t$ удовлетворяет неравенствам

$$
a\|g\|^{2} \leqslant \int_{-\infty}^{\infty} \int_{J} \frac{|\widehat{g}(x+i y)|^{2}}{K_{0}(x)} d \widetilde{u}^{\prime}(x) d y \leqslant A\|g\|^{2},
$$

где $а, A>0$ - абсолютные постоянные, т.е. не зависящие от функиии $g$ и веса $u$.

Для того чтобы преобразовать формулу (2) к нужному виду, введем две вспомогательные функции

$$
\eta(r)=\frac{e^{2 h(\varphi) r}}{K\left(r e^{i \varphi}\right)}, \quad u(r)=\frac{1}{2} \ln \frac{\eta(r)}{r^{4}} .
$$

С помошью этих функций получим представление

$$
\gamma^{\prime \prime}\left(e^{-i \varphi}(h(\varphi)-\xi)\right)=\int_{0}^{\infty} \frac{F\left(r e^{i \varphi}\right) e^{3 i \varphi} e^{h(\varphi) r}}{r^{2} K\left(r e^{i \varphi}\right)} e^{\xi r-2 u(r)} d r .
$$

Таким образом, мы сможем применить теорему В, если докажем выпуклость функции $u(t)$ на $\mathbb{R}_{+}$. С помощью отображения $z e^{i \varphi}-h(\varphi)=w$ преобразуем область $D$ в область $D_{\varphi}$, которая располагается на левой полуплоскости и касается оси ординат. Пусть

$$
D_{\varphi}=\left\{w=x+i y: f_{1}(x)<y<f_{2}(x),-R_{\varphi}<x<0\right\}
$$

и $f(x)=f_{2}(x)-f_{1}(x)$. Тогда $f$-неотрицательная вогнутая функция на отрезке $\left[-R_{\varphi}, 0\right]$. По определению имеем

$$
\begin{aligned}
K\left(r e^{i \varphi}\right) & =\int_{D} e^{2 r \operatorname{Re} z e^{i \varphi}} d v(z)=e^{2 h(\varphi) r} \int_{D_{\varphi}} e^{2 r x} d x d y \\
& =e^{2 h(\varphi) r} \int_{-R_{\varphi}}^{0} e^{2 r x} f(x) d x .
\end{aligned}
$$

Лемма 1. 1) Функиия $и(r)$ выпукла на $(0, \infty)$ и, более того,

$$
\frac{1}{2 r^{2}} \leqslant u^{\prime \prime}(r) \leqslant \frac{2}{r^{2}}, \quad r>0 .
$$

2) Если $\widetilde{u}(x)=\sup _{r>0}(x r-u(r))-$ функиия, сопряженная по Юнгу к функции и, то

$$
\frac{1}{2 x^{2}} \leqslant \widetilde{u}^{\prime \prime}(x) \leqslant \frac{8}{x^{2}}, \quad x<0 .
$$


ДоКАЗАТЕЛЬСТВО. Проводя непосредственные вычисления, получим представление

$$
u^{\prime \prime}(r)=\frac{4 a_{0}^{2}+a_{1}^{2}-a_{2} a_{0}}{2 a_{0}^{2} r^{2}},
$$

где

$$
a_{k}=\int_{-2 r R_{\varphi}}^{0} x^{k} e^{x} f\left(\frac{x}{2 r}\right) d x, \quad k=0,1,2 .
$$

Поскольку по неравенству Гёльдера $a_{1}^{2} \leqslant a_{0} a_{2}$, то

$$
u^{\prime \prime}(r) \leqslant \frac{2}{r^{2}} .
$$

Нижняя оценка для $u^{\prime \prime}(x)$ будет следовать из соотношения

$$
3 a_{0}^{2}+a_{1}^{2}-a_{0} a_{2} \geqslant 0 .
$$

Зафиксируем $r>0$, функцию $f\left(\frac{x}{2 r}\right)$ обозначим через $g(x)$. Тогда $g(x)$ - неотрицательная вогнутая функция на некотором интервале $(T, 0)$. По теореме о среднем значении для интеграла Римана существует число $t \in(T, 0)$ такое, что

$$
a_{2}=\int_{T}^{0} x^{2} e^{x} g(x) d x=t \int_{T}^{0} x e^{x} g(x) d x=t a_{1} .
$$

Если $t \geqslant-2 \sqrt{3}$, то $-a_{2}=-a_{1} t \geqslant 2 \sqrt{3} a_{1}$ и

$$
3 a_{0}^{2}+a_{1}^{2}-a_{0} a_{2} \geqslant\left(\sqrt{3} a_{0}\right)^{2}+a_{1}^{2}+2 \sqrt{3} a_{1} a_{0}=\left(\sqrt{3} a_{0}+a_{1}\right)^{2} \geqslant 0 .
$$

Докажем, что на самом деле $t>-3$. По определению числа $t$ имеем

$$
\int_{T}^{t}(x-t) x e^{x} g(x) d x=\int_{t}^{0}(t-x) x e^{x} g(x) d x .
$$

Положим $p=g(t)$; тогда в силу вогнутости $g(x)$ верны неравенства

$$
\begin{array}{ll}
g(x) \geqslant \frac{p}{t} x, & t \leqslant x \leqslant 0, \\
g(x) \leqslant \frac{p}{t} x, & T \leqslant x \leqslant t .
\end{array}
$$

Следовательно,

$$
\frac{p}{t} \int_{T}^{t}(x-t) x^{2} e^{x} d x \geqslant \frac{p}{t} \int_{t}^{0}(t-x) x^{2} e^{x} d x
$$

или

$$
\int_{T}^{t}(t-x) x^{2} e^{x} d x \geqslant \int_{t}^{0}(x-t) x^{2} e^{x} d x
$$

Вычислим интегралы и получим неравенство

$$
(t-T) T^{2} e^{T}+(3 T-2 t) T e^{T}+(2 t-6 T) e^{T}+6 e^{T} \leqslant 2 t+6 .
$$


Выражение, стоящее в левой части этого неравенства, положительное. Действительно, при фиксированном $T$ оно является линейной функцией от $t$. Вычислим ее значения при $t=0$ и $t=T$, получим соответственно $e^{T}\left(-T^{3}+3 T^{2}-6 T+6\right)>0$ и $e^{T}\left(T^{2}-4 T+6\right)>0$. Таким образом, $2 t+6>0$ и $t>-3$. Первое утверждение леммы доказано.

Докажем второе утверждение. Сначала выясним поведение функции $u(r)$ при $r \rightarrow \infty$. По формуле (4) имеем

$$
K\left(r e^{i \varphi}\right) \leqslant e^{2 h(\varphi) r}\left|D_{\varphi}\right|=e^{2 h(\varphi) r}|D|,
$$

где $|D|$-площадь области $D$. Чтобы оценить $K\left(r e^{i \varphi}\right)$ снизу, впишем в область $D_{\varphi}$ какой-либо треугольник $\triangle$ с вершиной $P$ на оси ординат и с основанием, параллельным оси $O y$. Пусть $h$ - высота этого треугольника и $a$ - длина основания. Для $r>\frac{1}{h}$ через $\triangle_{r}$ обозначим треугольник с высотой $\frac{1}{r}$, вершиной в $P$, подобньй треугольнику $\triangle$. Тогда

$$
K\left(r e^{i \varphi}\right) \geqslant e^{2 h(\varphi) r} \int_{\triangle_{r}} e^{2 x r} d x d y \geqslant e^{2 h(\varphi) r-2}\left|\triangle_{r}\right|=\frac{a e^{2 h(\varphi) r}}{2 e^{2} r^{2} h}
$$

Из этих двух оценок вытекает соотношение

$$
\frac{1}{2} \ln \frac{1}{|D| r^{4}} \leqslant u(r) \leqslant \frac{1}{2} \ln \frac{2 e^{2} h}{a r^{2}}, \quad r>\frac{1}{h} .
$$

Отсюда следует, что

$$
\lim _{r \rightarrow \infty} u(r)=-\infty, \quad\left|\frac{u(r)}{\ln r}\right|=O(1), \quad r \rightarrow \infty,
$$

и в силу выпуклости $u(r)$ получим, в частности, $\lim _{r \rightarrow \infty} u^{\prime}(r)=0$. Из утверждения 1) леммы теперь вытекает, что функция $u^{\prime}(r)$ отрицательна, строго возрастает и удовлетворяет неравенствам

$$
-\frac{2}{r} \leqslant u^{\prime}(r) \leqslant-\frac{1}{2 r}, \quad r>0 .
$$

Кроме того, очевидно, $\lim _{r \rightarrow 0} u(r)=+\infty$. Таким образом, функция $\widetilde{u}(x)$ определена для $x<0$, и поскольку при $x<0$

$$
\lim _{r \rightarrow 0+}(x r-u(r))=\lim _{r \rightarrow \infty}(x r-u(r))=-\infty,
$$

то супремум в определении $\widetilde{u}(x)$ достигается в единственной стационарной точке $r=r(x)$, в которой $u^{\prime}(r)=x$.

Из соотношения (5) получим

$$
-\frac{2}{r(x)} \leqslant x \leqslant-\frac{1}{2 r(x)}, \quad x<0
$$

или

$$
-\frac{1}{x} \leqslant r(x) \leqslant-\frac{2}{x}, \quad x<0
$$


По определению функции $\widetilde{u}(x)$ имеем тождество

$$
\widetilde{u}(x) \equiv x r(x)-u(r(x)), \quad x<0,
$$

или

$$
\widetilde{u}\left(u^{\prime}(r)\right) \equiv u^{\prime}(r) r-u(r), \quad r>0 .
$$

Продифференцируем дважды последнее тождество:

$$
\widetilde{u}^{\prime}\left(u^{\prime}(r)\right) \equiv r, \quad \widetilde{u}^{\prime \prime}\left(u^{\prime}(r)\right) u^{\prime \prime}(r) \equiv 1, \quad r>0 .
$$

Отсюда с учетом (6) и утверждения 1$)$ получим

$$
\widetilde{u}^{\prime \prime}(x) \equiv \frac{1}{u^{\prime \prime}(r(x))} \leqslant 2 r^{2}(x) \leqslant \frac{8}{x^{2}}, \quad x<0,
$$

и

$$
\widetilde{u}^{\prime \prime}(x) \geqslant \frac{1}{2} r^{2}(x) \geqslant \frac{1}{2 x^{2}}, \quad x<0 .
$$

Лемма доказана.

Мы обосновали применимость теоремы В для оценки $\gamma^{\prime \prime}$ по формуле (3). Вычислим функцию $K_{0}(x)$, входяшую в теорему $\mathrm{B}$, для данного случая. Пользуясь формулой (4) и определением функций $\eta(r)$ и $u(r)$, для $t<0$ получим

$$
\begin{aligned}
K_{0}(t, \varphi) & =\int_{0}^{\infty} \frac{e^{2 t r} r^{4}}{\eta(r)} d r=\int_{0}^{\infty} e^{2 t r} r^{4} K\left(r e^{i \varphi}\right) e^{-2 r h(\varphi)} d r \\
& =\int_{0}^{\infty} e^{2 t r} r^{4}\left(\int_{D_{\varphi}} e^{2 x r} d x d y\right) d r=\int_{D_{\varphi}}\left(\int_{0}^{\infty} e^{2 r(x+t)} r^{4} d r\right) d x d y .
\end{aligned}
$$

Отсюда вытекает представление

$$
K_{0}(t, \varphi)=\frac{3}{4} \int_{D_{\varphi}} \frac{1}{|x+t|^{5}} d x d y .
$$

Применим теорему В к функции

$$
g(r)=\frac{F\left(r e^{i \varphi}\right) e^{3 i \varphi} e^{h(\varphi) r}}{r^{2} K\left(r e^{i \varphi}\right)} .
$$

Тогда величина

$$
\|g\|^{2}=\int_{0}^{\infty}|g(r)|^{2} e^{-2 u(r)} d r=\int_{0}^{\infty} \frac{\left|F\left(r e^{i \varphi}\right)\right|^{2}}{K\left(r e^{i \varphi}\right)} d r=I_{\varphi}
$$

эквивалентна интегралу

$$
\int_{-\infty}^{\infty} \int_{-\infty}^{0} \frac{\left|\gamma^{\prime \prime}\left(e^{-i \varphi}(h(\varphi)-x-i y)\right)\right|^{2}}{K_{0}(x, \varphi)} d \widetilde{u}^{\prime}(x) d y .
$$

Из утверждения 2) леммы 1 получим

$$
\frac{a}{2} I_{\varphi} \leqslant \int_{-\infty}^{\infty} \int_{-\infty}^{0} \frac{\left|\gamma^{\prime \prime}\left(e^{-i \varphi}(h(\varphi)-x-i y)\right)\right|^{2}}{K_{0}(x, \varphi) x^{2}} d x d y \leqslant 8 A I_{\varphi},
$$

где $K_{0}(x, \varphi)$ определяется формулой $(7)$.

Приведем более детальное описание функции $K_{0}(t, \varphi)$. 
Лемма 2. Для любых $\varphi \in[0,2 \pi] u t<0$ имеют место соотношения

$$
\frac{s(t, \varphi)}{44|t|^{5}} \leqslant K_{0}(t, \varphi) \leqslant \frac{12}{11} \frac{s(t, \varphi)}{|t|^{5}},
$$

əде $s(t, \varphi)-$ площадь пересечения области $D_{\varphi}$ с полосой $\{z=x+i y: t<x<0\}$.

ДОКАЗАТЕЛЬСТВО. Пусть

$$
D_{\varphi}=\left\{w=x+i y: f_{1}(x)<y<f_{2}(x),-R_{\varphi}<x<0\right\}
$$

и $f(x)=f_{2}(x)-f_{1}(x)$. Тогда $f$ - неотрицательная вогнутая функция на отрезке $\left[-R_{\varphi}, 0\right]$ и из равенства (7) имеем

$$
K_{0}(t, \varphi)=\frac{3}{4} \int_{-R_{\varphi}}^{0} \frac{f(x) d x}{|x+t|^{5}} .
$$

Если $t \leqslant-R_{\varphi}$, то для $x$ из интервала интегрирования имеем $|t| \leqslant|x+t| \leqslant 2|t|$, поэтому

$$
\frac{3}{128|t|^{5}}\left|D_{\varphi}\right| \leqslant K_{0}(t, \varphi) \leqslant \frac{3}{4|t|^{5}} \int_{-R_{\varphi}}^{0} f(x) d x=\frac{3\left|D_{\varphi}\right|}{4|t|^{5}}
$$

и утверждение леммы верно, поскольку в этом случае $s(t, \varphi)=\left|D_{\varphi}\right|$. Пусть $t>-R_{\varphi}$ и $p=f(t)$. Из вогнутости функции $f$ следуют неравенства

$$
\begin{aligned}
f(x) & \leqslant \frac{p}{t} x, & -R_{\varphi} & \leqslant x \leqslant t, \\
f(x) & \geqslant \frac{p}{t} x, & t & \leqslant x \leqslant 0 .
\end{aligned}
$$

Поэтому

$$
\begin{gathered}
\int_{-R_{\varphi}}^{t} \frac{f(x) d x}{|x+t|^{5}} \leqslant \frac{p}{t} \int_{-R_{\varphi}}^{t} \frac{x d x}{|x+t|^{5}} \leqslant \frac{p}{|t|} \int_{-\infty}^{t} \frac{|x| d x}{(|x|+|t|)^{5}}=\frac{5 p}{192|t|^{4}} \\
\int_{t}^{0} \frac{f(x) d x}{|x+t|^{5}} \geqslant \frac{p}{t} \int_{t}^{0} \frac{x d x}{|x+t|^{5}}=\frac{11}{192|t|^{4}} .
\end{gathered}
$$

Таким образом,

$$
\int_{-R_{\varphi}}^{t} \frac{f(x) d x}{|x+t|^{5}} \leqslant \frac{5}{11} \int_{t}^{0} \frac{f(x) d x}{|x+t|^{5}}
$$

и

$$
K_{0}(t, \varphi)=\frac{3}{4} \int_{-R_{\varphi}}^{0} \frac{f(x) d x}{|x+t|^{5}} \leqslant \frac{48}{44} \int_{t}^{0} \frac{f(x) d x}{|x+t|^{5}} \leqslant \frac{12}{11|t|^{5}} s(t, \varphi) .
$$

Оценка снизу очевидна:

$$
K_{0}(t, \varphi)=\frac{3}{4} \int_{-R_{\varphi}}^{0} \frac{f(x) d x}{|x+t|^{5}} \geqslant \frac{3}{4} \int_{t}^{0} \frac{f(x) d x}{|x+t|^{5}} \geqslant \frac{1}{44|t|^{5}} s(t, \varphi) .
$$

Лемма доказана.

В заключение параграфа сформулируем в виде теоремы доказанные факты. 
ТЕОРема 1. Пусть $F=B(\gamma)$ - целая функиия, удовлетворяющая условию

$$
I_{\varphi}=\int_{0}^{\infty} \frac{\left|F\left(r e^{i \varphi}\right)\right|^{2}}{K\left(r e^{i \varphi}\right)} d r<\infty
$$

Тогда

$$
\frac{a I_{\varphi}}{88} \leqslant \int_{-\infty}^{\infty} \int_{-\infty}^{0} \frac{\left|\gamma^{\prime \prime}\left(e^{-i \varphi}(h(\varphi)-\xi)\right)\right|^{2}|x|^{3}}{s(x, \varphi)} d x d y \leqslant 9 A I_{\varphi}
$$

где $а, A>0$ - абсолютные постоянние из теоремь $\mathrm{B}$ и $s(x, \varphi)-$ площады пересечения области $D_{\varphi}$ с полосой $\{z: \operatorname{Re} z \in(x, 0)\}$.

\section{§2. Эквивалентность норм для функции $\gamma$}

По теореме 1 норма $\|F\|$ целой функции $F$, определенная в основной теореме, эквивалентна тройному интегралу

$$
\int_{0}^{2 \pi} \int_{-\infty}^{\infty} \int_{-\infty}^{0} \frac{\left|\gamma^{\prime \prime}\left(e^{-i \varphi}(h(\varphi)-\xi)\right)\right|^{2}|x|^{3}}{s(x, \varphi)} d x d y d \Delta(\varphi)
$$

В этом интеграле сделаем замену переменных

$$
\zeta=e^{-i \varphi}(h(\varphi)-x-i y), \quad \theta=\varphi
$$

Опишем геометрический смысл новых и прежних переменных.

Через $l(\varphi)$ далее будем обозначать направленную прямую $\left\{t e^{i \varphi}:-\infty<t<\infty\right\}$. На границе области $D$ выберем направление движения против часовой стрелки. Тем самым все касательные прямые к границе получают направление. Через $L(\varphi)$ обозначим касательную прямую, параллельную и сонаправленную с прямой $l\left(\frac{\pi}{2}-\varphi\right)$. Если $t e^{-i \varphi}$ - точка пересечения прямых $L(\varphi)$ и $l(-\varphi)$, то, как нетрудно проверить, $h(\varphi)=t$. Если заданы переменные $\varphi \in[0,2 \pi), x<0, y$, то $\zeta-$ точка плоскости, которая в системе координат, образованной прямыми $l(-\varphi)$ (ось абсцисс) и $l\left(\frac{\pi}{2}-\varphi\right)$ (ось ординат), имеет координаты $(h(\varphi)-x ;-y)$. При этом условие $x<0$ означает, что опорная прямая $L(\varphi)$ отделяет точку $\zeta$ от области $D$.

Определим область изменения переменных $\theta$ и $\zeta$. Точка $\zeta$, очевидно, лежит вне области $D$. При фиксированном $\zeta \in \mathbb{C} \backslash \bar{D}$ угол $\theta$ должен быть таким, чтобы опорная прямая $L(\theta)$ отделяла точку $\zeta$ от области $D$. Проведем две касательные прямые из точки $\zeta$ к гранище области $D$. Пусть они сонаправлены с прямыми $l\left(\varphi_{1}\right)$ и $l\left(\varphi_{2}\right)$, причем $0 \leqslant \varphi_{1} \leqslant \varphi_{2}$. Тогда угол $\theta$ меняется от $\varphi_{-}(\zeta)=\frac{\pi}{2}-\varphi_{2}$ до $\varphi_{+}(\zeta)=\frac{\pi}{2}-\varphi_{1}$. Якобиан перехода от переменных $\varphi, x, y$ к переменным $\zeta, \theta$ равен тождественно 1, и $x=h(\theta)-\operatorname{Re} \zeta e^{i \theta}$.

Таким образом,

$$
\begin{aligned}
\int_{0}^{2 \pi} \int_{-\infty}^{\infty} & \int_{-\infty}^{0} \frac{\left|\gamma^{\prime \prime}\left(e^{-i \varphi}(h(\varphi)-\xi)\right)\right|^{2}|x|^{3}}{s(x, \varphi)} d x d y d \Delta(\varphi) \\
& =\int_{\mathbb{C} \backslash \bar{D}}\left|\gamma^{\prime \prime}(\zeta)\right|^{2}\left(\int_{\varphi_{-}(\zeta)}^{\varphi_{+}(\zeta)} \frac{\left(\operatorname{Re} \zeta e^{i \theta}-h(\theta)\right)^{3}}{s\left(h(\theta)-\operatorname{Re} \zeta e^{i \theta}, \theta\right)} d \Delta(\theta)\right) d v(\zeta)
\end{aligned}
$$

Внутренний интеграл, стоящий в правой части равенства, обозначим через $p(\zeta)$. 
Теорема 1'. Пусть $F=B(\gamma)-$ челая функиия, удовлетворяющая условию

$$
\|F\|^{2}=\int_{0}^{2 \pi} \int_{0}^{\infty} \frac{\left|F\left(r e^{i \varphi}\right)\right|^{2}}{K\left(r e^{i \varphi}\right)} d r d \Delta(\varphi)<\infty
$$

$u$

$$
p(\zeta)=\int_{\varphi_{-}(\zeta)}^{\varphi_{+}(\zeta)} \frac{\left(\operatorname{Re} \zeta e^{i \theta}-h(\theta)\right)^{3}}{s\left(h(\theta)-\operatorname{Re} \zeta e^{i \theta}, \theta\right)} d \Delta(\theta)
$$

Тогда

$$
\frac{a}{88}\|F\|^{2} \leqslant \int_{\mathbb{C} \backslash \bar{D}}\left|\gamma^{\prime \prime}(\zeta)\right|^{2} p(\zeta) d v(\zeta) \leqslant 9 A\|F\|^{2} .
$$

Наша дальнейшая цель - показать, что данный интеграл от $\left|\gamma^{\prime \prime}\right|^{2}$ эквивалентен норме

$$
\|\gamma\|^{2}=\int_{\mathbb{C} \backslash \bar{D}}\left|\gamma^{\prime \prime}(\zeta)\right|^{2} d^{2}(\zeta) d v(\zeta)
$$

где $d(\zeta)=\inf _{z \in D}|\zeta-z|-$ расстояние от $\zeta$ до области $D$.

Докажем две геометрические леммы, введя предварительно необходимые обозначения. Через $s(x, \varphi)$ обозначим площадь части области

$$
D_{\varphi}=\left\{w=x+i y: f_{1}(x)<y<f_{2}(x),-R_{\varphi}<x<0\right\},
$$

отсекаемой прямой $\operatorname{Re} w=x$. Область $D_{\varphi}$ получается из области $D$ после преобразования $w=z e^{i \varphi}-h(\varphi)$. Тем самым $R_{\varphi}-$ расстояние между опорными прямыми $L(\varphi)$ и $L(\varphi+\pi)$. Через $l(x, \varphi)$ обозначим длину части границы $D_{\varphi}$, отсекаемой той же прямой, а через $u(x, \varphi)$ - длину хорды, отсекаемой областью $D_{\varphi}$ на этой прямой. Положим

$$
\sigma(D)=\inf _{\varphi \in[0,2 \pi]} R_{\varphi} .
$$

Ясно, что $\sigma(D)$ - некоторая характеристика области, причем ее можно оценить снизу следуюшим образом. Поскольку $R_{\varphi} R_{\varphi+\pi / 2}$ равно площади прямоугольника, содержащего область $D_{\varphi}$, то для любого $\varphi \in[0,2 \pi]$ имеем

$$
R_{\varphi} R_{\varphi+\pi / 2} \geqslant|D|,
$$

где $|D|-$ площадь области $D$. С другой стороны, очевидно, что $R_{\varphi} \leqslant \operatorname{diam}(D)$. Поэтому

$$
\sigma(D) \geqslant \frac{|D|}{\operatorname{diam}(D)}
$$

УТВеРЖДЕНИЕ 1. Через $A_{1}$ обозначим какую-нибудь точку касания опорной прямой $L(\varphi) \kappa$ области $D$, а через $A_{2}$ - точку касания опорной прямой $L(\varphi+\pi)$. Параллельно этим двум опорным прямым, ближе к прямой $L(\varphi)$, проведем прямую $L$ и через $B, C$ обозначим точки пересечения прямой $L$ с граничей области D. Угол $B A_{1} C$ обозначим через $\alpha(\varphi, L)$. Тогда для величиньь

$$
\alpha(D)=\inf _{\varphi \in[0,2 \pi], L} \alpha(\varphi, L)
$$

выполнена оченка

$$
\sin \frac{\alpha(D)}{2} \geqslant \frac{|D|}{6 \operatorname{diam}^{2}(D)} .
$$


ДоКАЗАТЕЛЬСТВО. При фиксированном $\varphi$ проведем прямую $L^{\prime}$, параллельную прямой $L$, точно посередине между прямыми $L(\varphi)$ и $L(\varphi+\pi)$. Если $B^{\prime}, C^{\prime}$ - точки пересечения прямой $L^{\prime}$ с границей области $D$, то в силу выпуклости области $D$ имеем $\angle B^{\prime} A_{1} C^{\prime} \leqslant \alpha(\varphi, L)$. Поскольку все углы $\alpha$ изменяются от 0 до $\pi$, то

$$
\sin \frac{\alpha(\varphi, L)}{2} \geqslant \sin \frac{\alpha\left(\varphi, L^{\prime}\right)}{2}
$$

Следовательно, в дальнейшем можно рассматривать только прямые $L$, проходяшие точно посередине между прямыми $L(\varphi)$ и $L(\varphi+\pi)$.

Через $B_{1}, C_{1}$ обозначим точки пересечения прямых $A_{2} B, A_{2} C$ с прямой $L(\varphi)$. Соответственно, пусть $B_{2}, C_{2}$ - точки пересечения прямых $A_{1} B, A_{1} C$ с прямой $L(\varphi+\pi)$. Далее, пусть $S_{i}, i=1,2,-$ площадь части $D$, лежащей в полосе между прямыми $L(\varphi), L$ и $L(\varphi+\pi), L$ соответственно. Тогда $S_{1}$ не превосходит площади трапеции $C C_{2} B_{2} B$. Из подобия треугольников $A_{2} B_{2} C_{2}$ и $A_{2} B C$ и из условия расположения прямой $L$ площадь этой трапеции равна утроенной плошади треугольника $A_{1} B C$ :

$$
S_{1} \leqslant 3 S_{\triangle A_{1} B C}
$$

Точно так же получим

$$
S_{2} \leqslant 3 S_{\triangle A_{2} B C}=3 S_{\triangle A_{1} B C}
$$

Таким образом,

$$
\begin{aligned}
|D| & =S_{1}+S_{2} \leqslant 6 S_{\triangle A_{1} B C} \\
& =3\left|A_{1} B\right|\left|A_{1} C\right| \sin \left(\angle B A_{1} C\right) \leqslant 3 \operatorname{diam}^{2}(D) \sin \left(\angle B A_{1} C\right) .
\end{aligned}
$$

Отсюда следует, что

$$
\sin \frac{\angle B A_{1} C}{2} \geqslant \frac{|D|}{6 \operatorname{diam}^{2}(D) \cos \left(\angle B A_{1} C / 2\right)} \geqslant \frac{|D|}{6 \operatorname{diam}^{2}(D)} .
$$

Утверждение доказано.

УТВЕРЖДЕНИЕ 2. Для всех $x \in\left(-\frac{\sigma}{2}, 0\right) u \varphi \in[0,2 \pi]$ выполнены следующие соотношения:

1) $\frac{1}{2}|x| u(x, \varphi) \leqslant s(x, \varphi) \leqslant \frac{3}{2}|x| u(x, \varphi)$;

2) $u(x, \varphi) \leqslant l(x, \varphi) \leqslant \frac{24 \operatorname{diam}^{4}(D)}{|D|^{2}} u(x, \varphi)$.

Eсли $x \leqslant-\frac{\sigma}{2}, m o$

$$
s(x, \varphi) \geqslant \frac{\sigma^{2}(D)}{4 \operatorname{diam}^{2}(D)}|D| .
$$


ДокАЗАТельСтво. Пусть $x \in\left(-\frac{\sigma}{2}, 0\right)$. Через $A_{2}$ обозначим точку касания (какую-нибудь) опорной прямой $L(\varphi+\pi)$. Пусть $B, C$ - точки пересечения прямой $L$, проведенной параллельно опорной прямой $L(\varphi)$ на расстоянии $|x|$ от нее, с границей области $D$. Через $B^{\prime}, C^{\prime}$ обозначим точки пересечения с $L(\varphi)$ прямых $A_{2} B, A_{2} C$ соответственно. Плошадь $s(x, \varphi)$ не превосходит плошади трапеции $B B^{\prime} C^{\prime} C$, поэтому

$$
s(x, \varphi) \leqslant|x| \frac{|B C|+\left|B^{\prime} C^{\prime}\right|}{2} \leqslant \frac{3}{2}|x||B C|=\frac{3}{2}|x| u(x, \varphi) .
$$

Левая часть двойного неравенства 1 ) получается еще проще: если $A_{1}$ - точка касания прямой $L(\varphi)$ с $D$, то

$$
s(x, \varphi) \geqslant S_{\triangle A_{1} B C}=\frac{1}{2}|x||B C|=\frac{1}{2}|x| u(x, \varphi) .
$$

Левая часть двойного неравенства 2) очевидна. Докажем правое неравенство. Используем при этом следуюшее тривиальное следствие теоремы косинусов: если $a, b, c$ - стороны треугольника и $\theta$ - угол между сторонами длины $b$ и $c$, то

$$
a \geqslant \sin \frac{\theta}{2}(b+c)
$$

По неравенству $(* *)$ имеем

$$
u(x, \varphi)=|B C| \geqslant \sin \frac{\angle B A_{1} C}{2}\left(\left|A_{1} B\right|+\left|A_{1} C\right|\right) .
$$

Воспользуемся утверждением 1:

$$
u(x, \varphi) \geqslant \frac{|D|}{6 \operatorname{diam}^{2}(D)}\left(\left|A_{1} B\right|+\left|A_{1} C\right|\right) .
$$

Для оценки снизу отрезков $\left|A_{1} B\right|$ и $\left|A_{1} C\right|$ мы намерены снова использовать неравенство $(* *)$, для чего необходимо оценить синусы $\angle A_{2} B^{\prime} C^{\prime}$ и $\angle A_{2} C^{\prime} B^{\prime}$. Из подобия треугольников $A_{2} B C$ и $A_{2} B^{\prime} C^{\prime}$ и из условия $|x| \leqslant \sigma(D) / 2 \leqslant R_{\varphi} / 2$ следует, что $\left|A_{2} B^{\prime}\right| \leqslant 2\left|A_{2} B\right|$ и $\left|A_{2} C^{\prime}\right| \leqslant 2\left|A_{2} C\right|$, значит, $\left|A_{2} B^{\prime}\right|,\left|A_{2} C^{\prime}\right| \leqslant 2 \operatorname{diam}(D)$. Высота треугольника $A_{2} B^{\prime} C^{\prime}$, опушенная из вершины $A_{2}$, равна $R_{\varphi}$ и не меньше, чем $\sigma(D)$. Таким образом, учитывая оценку (9), получим

$$
\sin \left(\angle A_{2} B^{\prime} C^{\prime}\right), \sin \left(\angle A_{2} C^{\prime} B^{\prime}\right) \geqslant \frac{\sigma(D)}{2 \operatorname{diam}(D)} \geqslant \frac{|D|}{2 \operatorname{diam}^{2}(D)} .
$$

Теперь на основе соотношения $(* *)$ имеем

$$
\begin{aligned}
& \left|A_{1} B\right| \geqslant \sin \frac{\angle A_{2} B^{\prime} C^{\prime}}{2}\left(\left|B B^{\prime}\right|+\left|A_{1} B^{\prime}\right|\right) \geqslant \frac{|D|}{4 \operatorname{diam}^{2}(D)}\left(\left|B B^{\prime}\right|+\left|A_{1} B^{\prime}\right|\right), \\
& \left|A_{1} C\right| \geqslant \sin \frac{\angle A_{2} C^{\prime} B^{\prime}}{2}\left(\left|C C^{\prime}\right|+\left|A_{1} C^{\prime}\right|\right) \geqslant \frac{|D|}{4 \operatorname{diam}^{2}(D)}\left(\left|C C^{\prime}\right|+\left|A_{1} C^{\prime}\right|\right) .
\end{aligned}
$$


В силу выпуклости области имеем $\left|B B^{\prime}\right|+\left|B^{\prime} C^{\prime}\right|+\left|C C^{\prime}\right| \geqslant l(x, \varphi)$, следовательно,

$$
\left|A_{1} B\right|+\left|A_{1} C\right| \geqslant \frac{|D|}{4 \operatorname{diam}^{2}(D)} l(x, \varphi) .
$$

Теперь можем завершить оценку (10):

$$
u(x, \varphi) \geqslant \frac{|D|^{2}}{24 \operatorname{diam}^{4}(D)} l(x, \varphi) .
$$

Нам осталось доказать последнее неравенство леммы. Если $x \leqslant-R_{\varphi}$, то $s(x, \varphi)=|D|$ и неравенство доказано. Предположим, что $x>-R_{\varphi}$. Через $A$ обозначим точку (какую-нибудь) касания опорной прямой $L(\varphi)$ с областью $D$. Пусть $B, C$ - точки пересечения прямой $L$, проведенной параллельно опорной прямой $L(\varphi)$ на расстоянии $|x|$ от нее, с гранищей области $D$. Через $B^{\prime}, C^{\prime}$ обозначим точки пересечения с $L(\varphi+\pi)$ прямых $A B, A C$ соответственно. Площадь $s(x, \varphi)$ не меньше площади треугольника $A B C$ :

$$
s(x, \varphi) \geqslant S_{\triangle A B C}
$$

Обозначим площадь части области $D$ между прямыми $L(\varphi+\pi)$ и $L$ через $S$. Тогда $S$ не превосходит площади трапеции $B B^{\prime} C^{\prime} C$. Пусть $k=|A B| /\left|A B^{\prime}\right|-$ коэффициент подобия треугольников $A B C$ и $A B^{\prime} C^{\prime}$. Условие $|x| \geqslant \sigma / 2$ влечет оценку

$$
k \geqslant \frac{\sigma}{2 \operatorname{diam}(D)}
$$

из которой следует оценка плошади трапеции:

$$
S_{B B^{\prime} C^{\prime} C} \leqslant\left(\frac{4 \operatorname{diam}^{2}(D)}{\sigma^{2}}-1\right) S_{\triangle A B C}
$$

Таким образом,

$$
S \leqslant\left(\frac{4 \operatorname{diam}^{2}(D)}{\sigma^{2}}-1\right) S_{\triangle A B C} \leqslant\left(\frac{4 \operatorname{diam}^{2}(D)}{\sigma^{2}}-1\right) s(x, \varphi)
$$

и

$$
|D|=S+s(x, \varphi) \leqslant \frac{4 \operatorname{diam}^{2}(D)}{\sigma^{2}} s(x, \varphi) .
$$

Утверждение доказано.

Напомним, что мы намеревались сравнить функцию

$$
p(\zeta)=\int_{\varphi_{-}(\zeta)}^{\varphi_{+}(\zeta)} \frac{\left(\operatorname{Re} \zeta e^{i \theta}-h(\theta)\right)^{3}}{s\left(h(\theta)-\operatorname{Re} \zeta e^{i \theta}, \theta\right)} d \Delta(\theta)
$$

с функцией расстояния $d(\zeta)$. 
Tеорема 2. Для точек $\zeta$ таких, что $d(\zeta) \leqslant \sigma(D) / 2$, определим функиию

$$
p_{0}(\zeta)=\int_{\varphi_{-}(\zeta)}^{\varphi_{+}(\zeta)} \frac{\left(\operatorname{Re} \zeta e^{i \theta}-h(\theta)\right)^{2}}{u\left(h(\theta)-\operatorname{Re} \zeta e^{i \theta}, \theta\right)} d \Delta(\theta)
$$

Тогда

$$
\frac{2}{3} p_{0}(\zeta) \leqslant p(\zeta) \leqslant 2 p_{0}(\zeta)
$$

Таким образом, для точек $\zeta$, близких к области D, достаточно оченить функщию $p_{0}(\zeta)$.

Eсли же $d(\zeta)>\sigma(D) / 2$, то через $I_{0}$ обозначим часть интервала $\left(\varphi_{-}(\zeta)\right.$, $\left.\varphi_{+}(\zeta)\right)$, әде выполнено условие

$$
\operatorname{Re} \zeta e^{i \varphi}-h(\varphi)>\frac{\sigma(D)}{2},
$$

и пусть I - остальная часть указанного интервала. Тогда

$$
p(\zeta) \leqslant \frac{4 \operatorname{diam}^{2}(D) l(D)}{\sigma^{2}(D)|D|} d^{3}(\zeta)+\int_{I} \frac{\left(\operatorname{Re} \zeta e^{i \theta}-h(\theta)\right)^{2}}{u\left(h(\theta)-\operatorname{Re} \zeta e^{i \theta}, \theta\right)} d \Delta(\theta),
$$

где $l(D)$ - длина гранищы $D$.

ДоКАЗАТЕЛЬСТво. Отметим, что по определению функции $h(\varphi)$ имеем

$$
\operatorname{Re} \zeta e^{i \varphi}-h(\varphi)=\min _{z \in \bar{D}}\left(\operatorname{Re} \zeta e^{i \varphi}-\operatorname{Re} z e^{i \varphi}\right) \leqslant \min _{z \in \bar{D}}|\zeta-z|=d(\zeta)
$$

поэтому для точек $\zeta$ таких, что выполнено условие $d(\zeta) \leqslant \sigma(D) / 2$, при любых $\varphi \in\left(\varphi_{-}(\zeta), \varphi_{+}(\zeta)\right)$ для оценки величины $s\left(h(\varphi)-\operatorname{Re} \zeta e^{i \varphi}, \varphi\right)$ можно применить утверждение 2 и немедленно получить оценки из первой части теоремы.

Если же интервал $I_{0}$ оказался непустым, то для $\theta \in I_{0}$ применим последнюю оценку в утверждении 2 :

$$
s\left(h(\theta)-\operatorname{Re} \zeta e^{i \theta}, \theta\right) \geqslant \frac{\sigma^{2}(D)|D|}{4 \operatorname{diam}^{2}(D)} .
$$

Отсюда, учитывая соотношение (11) и геометрический смысл функции $\Delta(\theta)$, получим последнюю нижнюю оценку в теореме. Теорема доказана.

Опишем интеграл, фигурирующий в определении функции $p_{0}(\zeta)$, в геометрических терминах. Интервал интегрирования $\left(\varphi_{-}(\zeta), \varphi_{+}(\zeta)\right)$ состоит из углов $\varphi$ таких, что $\operatorname{Re} \zeta e^{i \varphi}-h(\varphi) \geqslant 0$. Другими словами, это те направления $\varphi$, для которых опорная прямая $L(\varphi)$ отделяет точку $\zeta$ от области $D$. При этом величина $\operatorname{Re} \zeta e^{i \varphi}-h(\varphi)$ - это расстояние от точки $\zeta$ до опорной прямой $L(\varphi)$. Если эту опорную прямую перенесем параллельно на расстояние $\operatorname{Re} \zeta e^{i \varphi}-h(\varphi)$, то на полученной прямой область $D$ отсечет хорду, длину которой мы обозначили через $u\left(h(\varphi)-\operatorname{Re} \zeta e^{i \varphi}, \varphi\right)$. Наконец, геометрический смысл функции $\Delta(\varphi)$ заключается в том, что разность $\Delta\left(\varphi_{1}\right)-\Delta\left(\varphi_{2}\right)$ при $\varphi_{1} \geqslant \varphi_{2}$ равна длине дуги гранищы $D$ от точки касания опорной прямой $L\left(\varphi_{1}\right)$ до точки касания опорной прямой $L\left(\varphi_{2}\right)$ (см. [3]). 
Данное описание не связано с системой координат. Далее мы выберем систему координат, связанную с фиксированной точкой $\zeta$, область $D$ частично опишем как надграфик некоторой выпуклой функции $f$ и попробуем интеграл из определения функции $p_{0}(\zeta)$ записать с помощью функции $f$.

За начало координат возьмем точку $\zeta$. На гранище $D$ существует единственная точка $z_{0}$ такая, что

$$
d(\zeta)=\operatorname{dist}(\zeta)=\inf _{z \in \partial D}|z-\zeta|=\left|z_{0}-\zeta\right| .
$$

Ось ординат направим от точки $\zeta$ к точке $z_{0}$. В этой системе координат область $D$ является частью надграфика некоторой выпуклой функции $f(x)$, определенной на интервале $\left(X_{1}, X_{2}\right)$, где

$$
X_{1}=h(\pi), \quad X_{2}=h(0) .
$$

Углы рассматриваются, естественно, в новой системе координат. Углы наклона к оси абсцисс двух касательных к области $D$, проходящих через начало координат, обозначим через $\varphi_{1}$ и $\varphi_{2}$, причем $\varphi_{1} \leqslant \varphi_{2}$. Тогда интеграл из определения $p_{0}$ вычисляется в пределах от $\varphi_{-}=\frac{\pi}{2}-\varphi_{2}$ до $\varphi_{+}=\frac{\pi}{2}-\varphi_{1}$. Расстояние от точки $\zeta$ до области $D$ в этой системе координат выражается как $f(0)$ или $-h(\pi / 2)$.

Далее предположим, что гранища области $D$ не содержит углов и прямолинейных отрезков (см. замечание в конще введения). Для функции $f$ это значит, что производная $f^{\prime}$ является строго возрастающей непрерывной функцией.

Если переменная $\theta$ меняется от $\varphi_{-}$до $\varphi_{+}$, то величина $\frac{\pi}{2}-\theta$ монотонно меняется от $\varphi_{2}$ до $\varphi_{1}$, т. е. от угла наклона касательной к графику функции $f$ в точке $X_{1}$ до угла наклона касательной в точке $X_{2}$. Следовательно, если определим точку $x(\theta)$ из равенства

$$
f^{\prime}(x(\theta))=\operatorname{tg}\left(\frac{\pi}{2}-\theta\right)=\operatorname{ctg}(\theta),
$$

то точка $x(\theta)$ будет монотонно меняться от $X_{1}$ до $X_{2}$, причем точка $(x(\theta) ; f(x(\theta)))$ является точкой опоры опорной прямой $L(\theta)$. Из указанного выше геометрического смысла функции $\Delta(\theta)$ получим

$$
d \Delta(\theta)=d\left(\int^{x(\theta)} \sqrt{1+\left(f^{\prime}(s)\right)^{2}} d s\right)=\sqrt{1+\left(f^{\prime}(x(\theta))\right)^{2}} d x(\theta)=\frac{1}{|\sin \theta|} d x(\theta) .
$$

Для функции $p_{0}$ имеем следуюшее представление:

$$
p_{0}(\zeta)=\int_{\varphi_{-}}^{\varphi_{+}} \frac{h^{2}(\theta)}{u(h(\theta), \theta)} \frac{1}{|\sin \theta|} d x(\theta)
$$

где величины в интеграле правой части выражены в системе координат, связанной с точкой $\zeta$.

Далее нам необходимо представить выражение $u(h(\theta), \theta)$, используя функцию $f$. Для этого введем следуюшие функции. Рассмотрим любую точку $x_{0} \in\left[X_{1}, X_{2}\right]$ и положительное число $\delta$. Положим

$$
\begin{aligned}
& \rho_{+}\left(f, x_{0}, \delta\right)=\sup \left\{\rho: \rho \leqslant X_{2}-x_{0}, \int_{0}^{\rho}\left(f^{\prime}\left(x_{0}+y\right)-f^{\prime}\left(x_{0}\right)\right) d y \leqslant \delta\right\} \\
& \rho_{-}\left(f, x_{0}, \delta\right)=\sup \left\{\rho: \rho \leqslant x_{0}-X_{1}, \int_{0}^{\rho}\left(f^{\prime}\left(x_{0}\right)-f^{\prime}\left(x_{0}-y\right)\right) d y \leqslant \delta\right\} .
\end{aligned}
$$


Заметим, что если, например, $x_{0}+\rho_{+}<X_{2}$, то

$$
\int_{0}^{\rho_{+}}\left(f^{\prime}\left(x_{0}+y\right)-f^{\prime}\left(x_{0}\right)\right) d y=\delta
$$

Вычислив непосредственно интеграл, убеждаемся в том, что $x_{0}+\rho_{+}-$это точка, в которой значение функции $f$ ровно на $\delta$ превосходит значения касательной функции, определенной в точке $x_{0}$. Положим

$$
\widetilde{\rho}\left(f, x_{0}, \delta\right)=\rho_{-}\left(f, x_{0}, \delta\right)+\rho_{+}\left(f, x_{0}, \delta\right)
$$

Опорная прямая $L(\theta)$ - это касательная к графику функции $f(x)$ в точке $x(\theta)$, определяемой уравнением (12). Сдвиг опорной прямой $L(\theta)$ на расстояние $-h(\theta)$ в выбранной системе координат означает параллельньй перенос соответствующей касательной по вертикали вверх на $-h(\theta) /|\sin \theta|$. Если

$$
\begin{aligned}
& x(\theta)-\rho_{-}\left(f, x(\theta),-\frac{h(\theta)}{|\sin \theta|}\right)>X_{1}, \\
& x(\theta)+\rho_{+}\left(f, x(\theta),-\frac{h(\theta)}{|\sin \theta|}\right)<X_{2},
\end{aligned}
$$

то $\widetilde{\rho}(f, x(\theta),-h(\theta) /|\sin \theta|)$ есть не что иное, как длина проекции хорды сдвинутой опорной прямой (длину которой мы обозначали через $u(h(\theta), \theta)$ ) на ось абсцисс. Угол наклона опорной прямой $L(\theta)$ к оси абсцисс равен $\pi / 2-\theta$, поэтому

$$
u(h(\theta), \theta)=\frac{\widetilde{\rho}(f, x(\theta),-h(\theta) /|\sin \theta|)}{|\sin \theta|} .
$$

Допустим теперь, что

$$
\begin{aligned}
& x(\theta)-\rho_{-}\left(f, x(\theta),-\frac{h(\theta)}{|\sin \theta|}\right)>X_{1}, \\
& x(\theta)+\rho_{+}\left(f, x(\theta),-\frac{h(\theta)}{|\sin \theta|}\right)=X_{2} .
\end{aligned}
$$

Это может случиться, если

$$
f\left(X_{2}\right)-\left(f(x(\theta))+f^{\prime}(x(\theta))\left(X_{2}-x(\theta)\right)\right) \leqslant-\frac{h(\theta)}{|\sin \theta|} .
$$

Пусть точки пересечения сдвинутой опорной прямой $L(\theta)$ с границей области $D$ имеют абсциссы $x_{1}, x_{2}, X_{1}<x_{1}<x_{2}<X_{2}$. По утверждению 2 имеем

$$
u(h(\theta), \theta) \geqslant \frac{|D|^{2}}{24 \operatorname{diam}^{4}(D)} l(h(\theta), \theta),
$$

где $l(h(\theta), \theta)$ - длина дуги границы, отсекаемой сдвинутой опорной прямой $L(\theta)$. Эта длина, в свою очередь, больше длины отрезка, соединяющего точки $\left(x_{1} ; f\left(x_{1}\right)\right)$ и $\left(X_{2} ; f\left(X_{2}\right)\right)$, и, тем более, больше длины проекции этого отрезка на ось абсцисс. 
В данном случае длина проекции по определению и есть $\widetilde{\rho}(f, x(\theta),-h(\theta) /|\sin \theta|)$. Таким образом, имеем оценку

$$
u(h(\theta), \theta) \geqslant \frac{|D|^{2}}{24 \operatorname{diam}^{4}(D)|\sin \theta|} \widetilde{\rho}\left(f, x(\theta),-\frac{h(\theta)}{|\sin \theta|}\right) .
$$

Здесь мы воспользовались тем, что угол наклона к оси абсцисс отрезка, соединяющего точки $\left(x_{1} ; f\left(x_{1}\right)\right)$ и $\left(X_{2} ; f\left(X_{2}\right)\right)$, меньше, чем соответствующий угол для опорной прямой $L(\theta)$. Оценка сверху для величины $u(h(\theta), \theta)$ в этом случае очевидна:

$$
u(h(\theta), \theta) \leqslant \frac{\widetilde{\rho}(f, x(\theta),-h(\theta) /|\sin \theta|)}{|\sin \theta|} .
$$

Остальные два случая рассматриваются аналогично.

Сформулируем доказанные оценки в виде леммы.

Лемма 3. Если $\theta \in\left(\varphi_{-}, \varphi_{+}\right)$, то имеют место соотношения

$$
\begin{aligned}
\frac{|D|^{2}}{24 \operatorname{diam}^{4}(D)|\sin \theta|} \widetilde{\rho}(f, x(\theta) & \left.,-\frac{h(\theta)}{|\sin \theta|}\right) \\
& \leqslant u(h(\theta), \theta) \leqslant \frac{1}{|\sin \theta|} \widetilde{\rho}\left(f, x(\theta),-\frac{h(\theta)}{|\sin \theta|}\right) .
\end{aligned}
$$

Условие $\theta \in\left(\varphi_{-}, \varphi_{+}\right)$связано с тем, что при доказательстве мы применили утверждение 2 .

Для дальнейших оценок потребуются некоторые факты из теории выпуклых функций.

Пусть $f$ - ограниченная вьпуклая функция на отрезке $\left[X_{1}, X_{2}\right]$, и пусть при этом производная $f^{\prime}(x)$ строго возрастающая и непрерывная. Функция

$$
g(t)=\sup _{X_{1} \leqslant x \leqslant X_{2}}(x t-f(x)), \quad-\infty<t<\infty,
$$

называется сопряжсенной по Юнгу к функции $f$. Пусть $T_{1}=f^{\prime}\left(X_{1}\right), T_{2}=f^{\prime}\left(X_{2}\right)$. Если $t \in\left(T_{1}, T_{2}\right)$, то супремум в определении $g$ достигается в единственной стационарной точке $x=x(t)$, определяемой условием $f^{\prime}(x)=t$, т. е.

$$
g(t) \equiv x(t) t-f(x(t)), \quad t \in\left(T_{1}, T_{2}\right) .
$$

Продифференцируем это тождество по $t$ :

$$
g^{\prime}(t) \equiv x(t)
$$

Тем самым, имеем

$$
\begin{aligned}
f^{\prime}\left(g^{\prime}(t)\right) & \equiv t, \quad t \in\left(T_{1}, T_{2}\right), \\
g^{\prime}\left(f^{\prime}(x)\right) & \equiv x, \quad x \in\left(X_{1}, X_{2}\right) .
\end{aligned}
$$


Если $t \geqslant T_{2}$ или $t \leqslant T_{1}$, то стационарная точка в интервале $\left(X_{1}, X_{2}\right)$ не существует и супремум достигается в граничной точке $x=X_{2}$ или $x=X_{1}$. Поэтому

$$
\begin{aligned}
& g(t)=X_{2} t-f\left(X_{2}\right), \quad t \geqslant T_{2}, \\
& g(t)=X_{1} t-f\left(X_{1}\right), \quad t \leqslant T_{1},
\end{aligned}
$$

причем

$$
\begin{aligned}
& g^{\prime}(t) \equiv X_{2}, \quad t \geqslant T_{2}, \\
& g^{\prime}(t) \equiv X_{1}, \quad t \leqslant T_{1} .
\end{aligned}
$$

Следовательно,

$$
\begin{aligned}
& f^{\prime}\left(g^{\prime}(t)\right) \equiv f^{\prime}\left(X_{2}\right)=T_{2} \leqslant t, \quad t \geqslant T_{2}, \\
& f^{\prime}\left(g^{\prime}(t)\right) \equiv f^{\prime}\left(X_{1}\right)=T_{1} \geqslant t, \quad t \leqslant T_{1} .
\end{aligned}
$$

В частности, композиция $f^{\prime}\left(g^{\prime}(t)\right)$ определена для всех $t$.

Для выпуклой функции $g$ на $\mathbb{R}$ и любого положительного числа $\delta$ определим величину $\rho=\rho\left(g, t_{0}, \delta\right)$ из условия

$$
\rho=\sup \left\{s>0: \int_{-s}^{s}\left|g^{\prime}\left(t_{0}+t\right)-g^{\prime}\left(t_{0}\right)\right| d t \leqslant \delta\right\} .
$$

Вычислив интеграл, можно выписать эквивалентное определение:

$$
g\left(t_{0}+\rho\right)+g\left(t_{0}-\rho\right)-2 g\left(t_{0}\right)=\delta .
$$

Следуюшая лемма связывает величины $\widetilde{\rho}(f, x, \delta)$ и $\rho\left(g, f^{\prime}(x), \delta\right)$ для сопряженных по Юнгу функций $f, g$.

ЛЕмма 4. Пусть $f$ - ограниченная выпуклая функиия на отрезке $\left[X_{1}, X_{2}\right]$ со строго возрастающей непрерывной производной $f^{\prime}$. Функция $g$ - сопряженная по Юнгу с функиией $f$. Тогда для любого $x \in\left[X_{1}, X_{2}\right]$ выполнены соотношения

$$
\delta \leqslant \widetilde{\rho}(f, x, \delta) \rho\left(g, f^{\prime}(x), \delta\right) \leqslant 3 \delta .
$$

ДокАЗАТЕЛЬСТво. Для упрошения записи зафиксируем $x, \delta$ и введем обозначения

$$
t=f^{\prime}(x), \quad \widetilde{\rho}=\widetilde{\rho}(f, x, \delta), \quad \rho_{ \pm}=\rho_{ \pm}(f, x, \delta), \quad \rho=\rho\left(g, f^{\prime}(x), \delta\right) .
$$

Докажем левое неравенство.

1. Пусть сначала $x+\rho_{+}<X_{2}, x-\rho_{-}>X_{1}$.

a) Если при этом $x+\rho_{+} \leqslant g^{\prime}(t+\rho)$, то $f^{\prime}\left(x+\rho_{+}\right) \leqslant f^{\prime}\left(g^{\prime}(t+\rho)\right) \leqslant t+\rho$. Поэтому

$$
\delta=\int_{0}^{\rho_{+}}\left(f^{\prime}(x+y)-f^{\prime}(x)\right) d y \leqslant \int_{0}^{\rho_{+}}\left(f^{\prime}\left(x+\rho_{+}\right)-t\right) d y \leqslant \rho \rho_{+} \leqslant \rho \widetilde{\rho} .
$$


b) Если $x-\rho_{-} \geqslant g^{\prime}(t-\rho)$, то $f^{\prime}\left(x-\rho_{-}\right) \geqslant f^{\prime}\left(g^{\prime}(t-\rho)\right) \geqslant t-\rho$ и

$$
\delta=\int_{0}^{\rho_{-}}\left(f^{\prime}(x)-f^{\prime}(x-y)\right) d y \leqslant \int_{0}^{\rho_{-}}\left(t-f^{\prime}\left(x-\rho_{-}\right)\right) d y \leqslant \rho \rho_{-} \leqslant \rho \widetilde{\rho} .
$$

c) Если $x+\rho_{+} \geqslant g^{\prime}(t+\rho)$ и $x-\rho_{-} \leqslant g^{\prime}(t-\rho)$, то

$$
\begin{aligned}
\delta & =\int_{0}^{\rho}\left(g^{\prime}(t+\tau)-g^{\prime}(t)\right) d \tau+\int_{0}^{\rho}\left(g^{\prime}(t)-g^{\prime}(t-\tau)\right) d \tau \\
& \leqslant \int_{0}^{\rho}\left(g^{\prime}(t+\rho)-g^{\prime}(t)\right) d \tau+\int_{0}^{\rho}\left(g^{\prime}(t)-g^{\prime}(t-\rho)\right) d \tau \leqslant \rho_{+} \rho+\rho_{-} \rho=\widetilde{\rho} \rho .
\end{aligned}
$$

2. Допустим, что $x+\rho_{+}=X_{2}, x-\rho_{-}>X_{1}$. Тогда $x+\rho_{+} \geqslant g^{\prime}(t+\rho)$. Если при этом $x-\rho_{-} \geqslant g^{\prime}(t-\rho)$, требуемую оценку получим так же, как в п. b). Если $x-\rho_{-} \leqslant g^{\prime}(t-\rho)$, то приходим к п. с).

Случай, когда $x-\rho_{-}=X_{1}, x+\rho_{+}<X_{2}$, рассматривается аналогично п. 2.

Последний возможньй случай, когда $x-\rho_{-}=X_{1}$ и $x+\rho_{+}=X_{2}$, включается в п. с).

Нижняя оценка леммы доказана.

Перейдем к верхним оценкам. Предварительно докажем вспомогательное неравенство

$$
\int_{0}^{\rho_{+}}\left(f^{\prime}(x+y)-f^{\prime}(x)\right) d y+\int_{0}^{\rho}\left(g^{\prime}(t+\tau)-g^{\prime}(t)\right) d \tau \geqslant \rho \rho_{+} .
$$

Пусть $x+\rho_{+}=X_{2}$ или $x+\rho_{+}<X_{2}$, но при этом $f^{\prime}\left(x+\rho_{+}\right) \geqslant t+\rho$. В обоих случаях имеем $x+\rho_{+} \geqslant g^{\prime}(t+\rho)$, поэтому

$$
\begin{aligned}
\int_{0}^{\rho_{+}}\left(f^{\prime}(x+y)-f^{\prime}(x)\right) d y \geqslant & \int_{0}^{g^{\prime}(t+\rho)-x}\left(f^{\prime}(x+y)-f^{\prime}(x)\right) d y \\
& +\int_{g^{\prime}(t+\rho)-x}^{\rho_{+}}\left(f^{\prime}\left(g^{\prime}(t+\rho)\right)-f^{\prime}(x)\right) d y .
\end{aligned}
$$

В интеграле, стоящем в левой части этого неравенства, произведем замену переменных $\tau=f^{\prime}(x+y)-t, y=g^{\prime}(\tau+t)-x$ :

$$
\begin{aligned}
\int_{0}^{\rho_{+}}\left(f^{\prime}(x+y)-f^{\prime}(x)\right) d y \geqslant & \int_{0}^{\rho} \tau d\left(g^{\prime}(\tau+t)-g^{\prime}(t)\right) \\
& +\int_{g^{\prime}(t+\rho)-x}^{\rho_{+}}\left(f^{\prime}\left(g^{\prime}(t+\rho)\right)-f^{\prime}(x)\right) d y .
\end{aligned}
$$

Применяя формулу интегрирования по частям, получим

$$
\begin{aligned}
\int_{0}^{\rho_{+}}\left(f^{\prime}(x+y)-f^{\prime}(x)\right) d y \\
\quad \geqslant \rho\left(g^{\prime}(t+\rho)-x\right)-\int_{0}^{\rho}\left(g^{\prime}(\tau+t)-g^{\prime}(t)\right) d \tau+\rho \rho_{+}+\rho\left(x-g^{\prime}(t+\rho)\right) \\
\quad=\rho \rho_{+}-\int_{0}^{\rho}\left(g^{\prime}(\tau+t)-g^{\prime}(t)\right) d \tau,
\end{aligned}
$$

и, тем самым, соотношение (16) для этого случая доказано. 
Пусть теперь $x+\rho_{+}<X_{2}$ и $f^{\prime}\left(x+\rho_{+}\right)<t+\rho$. Тогда

$$
\begin{aligned}
\int_{0}^{\rho}\left(g^{\prime}(\tau+t)-g^{\prime}(t)\right) d \tau \geqslant & \int_{0}^{f^{\prime}\left(x+\rho_{+}\right)-t}\left(g^{\prime}(\tau+t)-g^{\prime}(t)\right) d \tau \\
& +\int_{f^{\prime}\left(x+\rho_{+}\right)-t}^{\rho}\left(g^{\prime}\left(f^{\prime}\left(x+\rho_{+}\right)\right)-g^{\prime}(t)\right) d \tau
\end{aligned}
$$

В первом интеграле сделаем замену переменных $y=g^{\prime}(\tau+t)-g^{\prime}(t), \tau=f^{\prime}(y+x)-t$ и применим формулу интегрирования по частям:

$$
\begin{aligned}
\int_{0}^{\rho}\left(g^{\prime}(\tau+t)-g^{\prime}(t)\right) d \tau \geqslant \int_{0}^{\rho_{+}} y d\left(f^{\prime}(x+y)-f^{\prime}(x)\right)+\rho_{+}\left(\rho-f^{\prime}\left(x+\rho_{+}\right)+t\right) \\
=\rho_{+}\left(f^{\prime}\left(x+\rho_{+}\right)-f^{\prime}(x)\right) \\
\quad-\int_{0}^{\rho_{+}}\left(f^{\prime}(x+y)-f^{\prime}(x)\right) d y+\rho_{+} \rho-\rho_{+}\left(f^{\prime}\left(x+\rho_{+}\right)-f^{\prime}(x)\right) \\
=\rho_{+} \rho-\int_{0}^{\rho_{+}}\left(f^{\prime}(x+y)-f^{\prime}(x)\right) d y
\end{aligned}
$$

и в этом случае снова доказано соотношение (16).

Аналогичным образом доказывается соотношение

$$
\int_{0}^{\rho_{-}}\left(f^{\prime}(x)-f^{\prime}(x-y)\right) d y+\int_{0}^{\rho}\left(g^{\prime}(t)-g^{\prime}(t-\tau)\right) d \tau \geqslant \rho \rho_{-}
$$

Суммируя это неравенство с (16) и учитьвая определения величин $\rho, \rho_{ \pm}, \widetilde{\rho}$, получим

$$
3 \delta \geqslant \rho \widetilde{\rho} .
$$

Лемма доказана.

Теорема 2 , леммы 3 и 4 с учетом определения (12) точки $x(\theta)$ приводят к следующей теореме.

ТЕОРема 3. Для точек $\zeta$ таких, что $d(\zeta) \leqslant \sigma(D) / 2$, выполнень неравенства

$$
\begin{aligned}
& \frac{2}{9} \int_{\varphi_{-}}^{\varphi_{+}}|h(\theta) \sin \theta| \rho\left(g, \operatorname{ctg} \theta, \frac{|h(\theta)|}{|\sin \theta|}\right) d g^{\prime}(\operatorname{ctg} \theta) \\
& \quad \leqslant p(\zeta) \leqslant \frac{48 \operatorname{diam}^{4}(D)}{|D|^{2}} \int_{\varphi_{-}}^{\varphi_{+}}|h(\theta) \sin \theta| \rho\left(g, \operatorname{ctg} \theta, \frac{|h(\theta)|}{|\sin \theta|}\right) d g^{\prime}(\operatorname{ctg} \theta) .
\end{aligned}
$$

Ecли $d(\zeta)>\sigma(D) / 2$, то через $I_{0}$ обозначим часть интервала $\left(\varphi_{-}, \varphi_{+}\right)$, где выполнено условие

$$
-h(\theta)>\frac{\sigma(D)}{2}
$$


и пусть I - остальная часть указанного интервала. Тогда

$$
\begin{aligned}
p(\zeta) \leqslant & \frac{4 \operatorname{diam}^{2}(D) l(D)}{\sigma^{2}(D)|D|} d^{3}(\zeta) \\
& +\frac{48 \operatorname{diam}^{4}(D)}{|D|^{2}} \int_{I}|h(\theta) \sin \theta| \rho\left(g, \operatorname{ctg} \theta, \frac{|h(\theta)|}{|\sin \theta|}\right) d g^{\prime}(\operatorname{ctg} \theta),
\end{aligned}
$$

әде $l(D)-$ длина границь D.

В интегралах использована система координат, связанная с точкой $\zeta$, причем начало этой системы координат помещено в саму точку $\zeta$, а ось ординат направлена от точки $\zeta$ к точке $z_{0}$, определяемой из условия $d(\zeta)=\left|z_{0}-\zeta\right|$. В этой системе координат область $D$ оказывается частью надграфика некоторой ограниченной выпуклой функции $f(x), x \in\left[X_{1}, X_{2}\right]$. Если через $\theta_{-}, \theta_{+},-\pi / 2<\theta_{-}<$ $\theta_{+}<\pi / 2$, обозначим углы наклона к оси абсцисс касательных к графику функции $f(x)$, проходящих через начало координат, то $\varphi_{-}=\pi / 2-\theta_{+}, \varphi_{+}=\pi / 2-\theta_{-}$. Положим $\varphi=\pi / 2-\theta$. Тогда $\varphi \in\left(\theta_{-}, \theta_{+}\right)$и нам предстоит оценить функцию

$$
p_{1}(\zeta)=\int_{\theta_{-}}^{\theta_{+}}\left|h\left(\frac{\pi}{2}-\varphi\right) \cos \varphi\right| \rho\left(g, \operatorname{tg} \varphi, \frac{\left|h\left(\frac{\pi}{2}-\varphi\right)\right|}{\cos \varphi}\right) d g^{\prime}(\operatorname{tg} \varphi) .
$$

В этой системе координат расстояние $d(\zeta)$ равно $-h\left(\frac{\pi}{2}\right)$. Обозначим это расстояние через $d$. Определим точку $x=x(\varphi) \in\left[X_{1}, X_{2}\right]$ из условия

$$
f^{\prime}(x(\varphi))=\operatorname{tg} \varphi
$$

Тогда опорная прямая $L(\varphi)$ к области $D$ есть касательная к функции $f(x)$ в точке $x(\varphi)$, a $-h\left(\frac{\pi}{2}-\varphi\right)$ - это расстояние от этой касательной до начала координат. Легко видеть, что при этом число $-h\left(\frac{\pi}{2}-\varphi\right) / \cos \varphi$ равно ординате точки пересечения опорной прямой с осью ординат. Уравнение касательной, проведенной в точке $x(\varphi)$, имеет вид

$$
y=\operatorname{tg} \varphi(x-x(\varphi))+f(x(\varphi))
$$

поэтому

$$
-\frac{h\left(\frac{\pi}{2}-\varphi\right)}{\cos \varphi}=f(x(\varphi))-\operatorname{tg} \varphi x(\varphi) .
$$

Учитывая соотношения (14) и (18), получим

$$
-\frac{h\left(\frac{\pi}{2}-\varphi\right)}{\cos \varphi}=-g(\operatorname{tg} \varphi)
$$

Таким образом, когда $\varphi$ монотонно возрастает от 0 до $\theta_{+}$или убьвает от 0 до $\theta_{-}$, значение $-g(\operatorname{tg} \varphi)$ монотонно убывает от $d$ до 0 . Положим $\varphi_{0}=0$ и определим углы $\varphi_{n}$ из условий

$$
-\frac{h\left(\frac{\pi}{2}-\varphi_{n}\right)}{\cos \varphi_{n}}=\frac{d}{2^{|n|}},
$$

или, что то же самое, из равенств $-g\left(\operatorname{tg} \varphi_{n}\right)=2^{-|n|} d$. 
Интервал интегрирования разбивается на интервалы $\left(\varphi_{n}, \varphi_{n+1}\right], n \in \mathbb{Z}$, и сам интеграл в (17) может быть представлен в виде суммы интегралов по этим интервалам. Заметим, что $\rho(g, t, \delta)$ - неубывающая величина по переменной $\delta$, поэтому при $\varphi \in\left(\varphi_{n}, \varphi_{n+1}\right]$ имеем

$$
\begin{aligned}
\rho\left(g, \operatorname{tg} \varphi, 2^{-n-1} d\right) \leqslant \rho\left(g, \operatorname{tg} \varphi,-\frac{h\left(\frac{\pi}{2}-\varphi\right)}{\cos \varphi}\right) \leqslant \rho\left(g, \operatorname{tg} \varphi, 2^{-n} d\right), & n \geqslant 0, \\
\rho\left(g, \operatorname{tg} \varphi, 2^{-|n|} d\right) \leqslant \rho\left(g, \operatorname{tg} \varphi,-\frac{h\left(\frac{\pi}{2}-\varphi\right)}{\cos \varphi}\right) \leqslant \rho\left(g, \operatorname{tg} \varphi, 2^{-|n|+1} d\right), & n<0 .
\end{aligned}
$$

Таким образом, после замены переменных $t=\operatorname{tg} \varphi$ получаем

$$
\begin{aligned}
p_{1}(\zeta) \leqslant & \sum_{n=0}^{\infty} \frac{d}{2^{n}} \int_{\operatorname{tg} \varphi_{n}}^{\operatorname{tg} \varphi_{n+1}} \rho\left(g, t, \frac{d}{2^{n}}\right) d g^{\prime}(t) \\
& +\sum_{n=-1}^{\infty} \frac{d}{2^{|n|-1}} \int_{\operatorname{tg} \varphi_{n}}^{\operatorname{tg} \varphi_{n+1}} \rho\left(g, t, \frac{d}{2^{|n|-1}}\right) d g^{\prime}(t), \\
p_{1}(\zeta) \geqslant & \frac{d}{2} \int_{\operatorname{tg} \varphi_{-1}}^{\operatorname{tg} \varphi_{1}} \frac{1}{1+t^{2}} \rho\left(g, t, \frac{d}{2}\right) d g^{\prime}(t) .
\end{aligned}
$$

Получим верхние оценки для $p_{1}(\zeta)$. Положим

$$
t_{n}=\frac{\operatorname{tg} \varphi_{n}+\operatorname{tg} \varphi_{n+1}}{2} .
$$

Тогда при $n \geqslant 0$, очевидно, $g\left(t_{n}\right) \geqslant g\left(\operatorname{tg} \varphi_{n}\right)=-d / 2^{n}$, а при $n<0$ имеем $g\left(t_{n}\right) \geqslant g\left(\operatorname{tg} \varphi_{n+1}\right)=-d / 2^{|n|-1}$. Поэтому

$$
\begin{gathered}
g\left(\operatorname{tg} \varphi_{n}\right)+g\left(\operatorname{tg} \varphi_{n+1}\right)-2 g\left(t_{n}\right) \leqslant-\frac{d}{2^{n+1}}-\frac{d}{2^{n}}+\frac{2 d}{2^{n}}=\frac{d}{2^{n+1}}<\frac{d}{2^{n}}, \quad n \geqslant 0, \\
g\left(\operatorname{tg} \varphi_{n}\right)+g\left(\operatorname{tg} \varphi_{n+1}\right)-2 g\left(t_{n}\right) \leqslant \frac{d}{2^{|n|}}<\frac{d}{2^{|n|-1}}, \quad n \leqslant 0 .
\end{gathered}
$$

Сравнив эти оценки с определением (15) величины $\rho(g, t, \delta)$, получим

$$
\operatorname{tg} \varphi_{n+1}-t_{n}=t_{n}-\operatorname{tg} \varphi_{n}< \begin{cases}\rho\left(g, t_{n}, \frac{d}{2^{n}}\right), & n \geqslant 0, \\ \rho\left(g, t_{n}, \frac{d}{2^{|n|-1}}\right), & n<0 .\end{cases}
$$

Введем обозначения

$$
\rho_{n}= \begin{cases}\rho\left(g, t_{n}, \frac{d}{2^{n}}\right), & n \geqslant 0 \\ \rho\left(g, t_{n}, \frac{d}{2^{|n|-1}}\right), & n<0 .\end{cases}
$$

Теперь из соотношения $\left(19^{\prime}\right)$ получаем оценку

$$
\begin{aligned}
p_{1}(\zeta) \leqslant & \sum_{0}^{\infty} \frac{d}{2^{n}} \int_{t_{n}-\rho_{n}}^{t_{n}+\rho_{n}} \rho\left(g, t, \frac{d}{2^{n}}\right) d g^{\prime}(t) \\
& +\sum_{-1}^{-\infty} \frac{d}{2^{|n|-1}} \int_{t_{n}-\rho_{n}}^{t_{n}+\rho_{n}} \rho\left(g, t, \frac{d}{2^{|n|-1}}\right) d g^{\prime}(t) .
\end{aligned}
$$

Для продолжения доказательства теоремы сформулируем и докажем одну общую лемму. 
Лемма 5. Пусть $g$ - выпуклая функиия на $\mathbb{R} u \delta>0$. Для сокращения обозначений положим $\rho(t)=\rho(g, t, \delta)$. Имеют место утверждения:

1) ддя всех $t, \tau \in \mathbb{R}$

$$
|\rho(t)-\rho(\tau)| \leqslant|t-\tau|
$$

2) для любого $\tau$ выполнено

$$
\rho(t) \leqslant\left\{\begin{array}{lll}
\tau+\rho(\tau)+\rho(\tau+\rho(\tau))-t, & \text { если } & \tau \leqslant t \leqslant \tau+\rho(\tau), \\
t-\tau+\rho(\tau)+\rho(\tau-\rho(\tau)), & \text { если } & \tau-\rho(\tau) \leqslant t \leqslant \tau
\end{array}\right.
$$

3) для любого $t$ выполнено

$$
\delta \leqslant \int_{\tau-\rho(\tau)}^{\tau+\rho(\tau)} \rho(t) d g^{\prime}(t) \leqslant 4 \delta
$$

ДоказАТЕЛЬСтво. 1) Пусть $t \in[\tau, \tau+\rho(\tau)]$. Тогда для любого $y$ имеем $t+y \geqslant \tau+(y-t+\tau)$ и в силу монотонности функции $g^{\prime}$ получим

$$
\begin{aligned}
\int_{0}^{t+\rho(\tau)-\tau}\left(g^{\prime}(t+y)-g^{\prime}(t-y)\right) d y \\
\geqslant \int_{t-\tau}^{t+\rho(\tau)-\tau}\left(g^{\prime}(\tau+(y-t+\tau))-g^{\prime}(\tau-(y-t+\tau))\right) d y .
\end{aligned}
$$

После замены переменных $x=y-t+\tau$ в последнем интеграле получим

$$
\int_{0}^{t+\rho(\tau)-\tau}\left(g^{\prime}(t+y)-g^{\prime}(t-y)\right) d y \geqslant \int_{0}^{\rho(\tau)}\left(g^{\prime}(\tau+x)-g^{\prime}(\tau-x)\right) d x=\delta .
$$

Следовательно, $t+\rho(\tau)-\tau \geqslant \rho(t)$ или

$$
\rho(t)-\rho(\tau) \leqslant|t-\tau| .
$$

Аналогично, при $t \geqslant \tau$ для любого $y$ имеем $t-y \geqslant \tau-(t+y-\tau)$, т. е. $-g^{\prime}(t-y) \leqslant$ $-g^{\prime}(\tau-(t+y-\tau))$. Поэтому из неравенства

$$
\begin{aligned}
& \int_{0}^{\tau+\rho(\tau)-t}\left(g^{\prime}(t+y)-g^{\prime}(t-y)\right) d y \\
& \quad \leqslant \int_{0}^{\tau+\rho(\tau)-t}\left(g^{\prime}(\tau+(y+t-\tau))-g^{\prime}(\tau-(y+t-\tau))\right) d y
\end{aligned}
$$

заменой переменных $x=t+y-\tau$ получим оценку

$$
\int_{0}^{\tau+\rho(\tau)-t}\left(g^{\prime}(t+y)-g^{\prime}(t-y)\right) d y \leqslant \int_{t-\tau}^{\rho(\tau)}\left(g^{\prime}(t+x)-g^{\prime}(t-x)\right) d x \leqslant \delta
$$

и, следовательно, $\tau+\rho(\tau)-t \leqslant \rho(t)$, т. е.

$$
\rho(\tau)-\rho(t) \leqslant|t-\tau| .
$$


Вместе с неравенством (20) это значит, что выполнено соотношение

$$
|\rho(\tau)-\rho(t)| \leqslant|t-\tau|, \text { если } \tau \leqslant t \leqslant \tau+\rho(\tau) .
$$

В силу симметричности величины $\rho$ это соотношение точно так же может быть доказано для случая, когда $\tau \geqslant t \geqslant \tau-\rho(\tau)$. Наконец, в силу локальности свойства липшицевости оно выполняется для всех $\tau, t \in \mathbb{R}$.

2 ) Для краткости используем обозначения $a=\tau-\rho(\tau), b=\tau+\rho(\tau)$. Пусть $\tau \leqslant t \leqslant b$. Тогда $t-x \leqslant b-(t+x-b)$ и

$$
\int_{0}^{b+\rho(b)-t}\left(g^{\prime}(t+x)-g^{\prime}(t-x)\right) d x \geqslant \int_{b-t}^{b-t+\rho(b)}\left(g^{\prime}(b+(t+x-b))-g^{\prime}(b-(t+x-b))\right) d x .
$$

Замена переменных $y=t+x-b$ приводит к оценке

$$
\int_{0}^{b+\rho(b)-t}\left(g^{\prime}(t+x)-g^{\prime}(t-x)\right) d x \geqslant \int_{0}^{\rho(b)}\left(g^{\prime}(b+y)-g^{\prime}(b-y)\right) d y=\delta
$$

из которой следует требуемое неравенство $b+\rho(b)-t \geqslant \rho(t)$. Случай, когда $t \in[a, \tau]$ рассматривается аналогично.

3) Докажем сначала нижнюю оценку. Из утверждения п. 1) следуют неравенства

$$
\rho(t) \geqslant\left\{\begin{array}{l}
b-t, \text { если } \tau \leqslant t, \\
t-a, \text { если } t \leqslant \tau .
\end{array}\right.
$$

Поэтому

$$
\int_{a}^{b} \rho(t) d g^{\prime}(t) \geqslant \int_{a}^{\tau}(t-a) d\left(g^{\prime}(t)-g^{\prime}(\tau)\right)+\int_{\tau}^{b}(b-t) d\left(g^{\prime}(t)-g^{\prime}(\tau)\right) .
$$

Применим к последним интегралам формулу интегрирования по частям:

$$
\begin{aligned}
\int_{a}^{b} \rho(t) d g^{\prime}(t) & \geqslant \int_{a}^{\tau}\left(g^{\prime}(\tau)-g^{\prime}(t)\right) d t+\int_{\tau}^{b}\left(g^{\prime}(t)-g^{\prime}(\tau)\right) d t \\
& =\int_{\tau-\rho(\tau)}^{\tau+\rho(\tau)}\left|g^{\prime}(\tau)-g^{\prime}(t)\right| d t=\delta
\end{aligned}
$$

Нижняя оценка доказана.

Докажем верхнюю оценку. Из утверждения п. 2) следует

$$
\int_{a}^{b} \rho(t) d g^{\prime}(t) \leqslant \int_{a}^{\tau}(t-a+\rho(a)) d\left(g^{\prime}(t)-g^{\prime}(\tau)\right)+\int_{\tau}^{b}(b+\rho(b)-t) d\left(g^{\prime}(t)-g^{\prime}(\tau)\right) .
$$

Применяя формулу интегрирования по частям к последним интегралам, получим

$$
\begin{aligned}
\int_{a}^{b} \rho(t) d g^{\prime}(t) & \leqslant \rho(a)\left(g^{\prime}(\tau)-g^{\prime}(a)\right)+\rho(b)\left(g^{\prime}(b)-g^{\prime}(\tau)\right)+\int_{a}^{b}\left|g^{\prime}(t)-g^{\prime}(\tau)\right| d t \\
& =\rho(a)\left(g^{\prime}(\tau)-g^{\prime}(a)\right)+\rho(b)\left(g^{\prime}(b)-g^{\prime}(\tau)\right)+\delta
\end{aligned}
$$


Оценим первое слагаемое в правой части. Если $\tau \geqslant a+\rho(a)$, то

$$
\begin{aligned}
\rho(a)\left(g^{\prime}(\tau)-g^{\prime}(a)\right) & =\int_{a}^{a+\rho(a)}\left(g^{\prime}(\tau)-g^{\prime}(x)\right) d x+\int_{a}^{a+\rho(a)}\left(g^{\prime}(x)-g^{\prime}(a)\right) d x \\
& \leqslant \int_{a}^{\tau}\left(g^{\prime}(\tau)-g^{\prime}(x)\right) d x+\delta .
\end{aligned}
$$

Если же $\tau \leqslant a+\rho(a)$, то

$$
\begin{aligned}
\rho(a) & \left(g^{\prime}(\tau)-g^{\prime}(a)\right)=\int_{a}^{a+\rho(a)}\left(g^{\prime}(\tau)-g^{\prime}(a)\right) d x \\
& =\int_{a}^{\tau}\left(g^{\prime}(\tau)-g^{\prime}(x)\right) d x+\int_{a}^{\tau}\left(g^{\prime}(x)-g^{\prime}(a)\right) d x+\int_{\tau}^{a+\rho(a)}\left(g^{\prime}(\tau)-g^{\prime}(a)\right) d x \\
& \leqslant \int_{a}^{a+\rho(a)}\left(g^{\prime}(x)-g^{\prime}(a)\right) d x+\int_{a}^{\tau}\left(g^{\prime}(\tau)-g^{\prime}(x)\right) d x \\
& \leqslant \delta+\int_{a}^{\tau}\left(g^{\prime}(\tau)-g^{\prime}(x)\right) d x
\end{aligned}
$$

Таким образом, в любом случае имеем оценку

$$
\rho(a)\left(g^{\prime}(\tau)-g^{\prime}(a)\right) \leqslant \delta+\int_{a}^{\tau}\left(g^{\prime}(\tau)-g^{\prime}(x)\right) d x .
$$

Аналогично получается оценка

$$
\rho(b)\left(g^{\prime}(b)-g^{\prime}(\tau)\right) \leqslant \delta+\int_{\tau}^{b}\left(g^{\prime}(x)-g^{\prime}(\tau)\right) d x .
$$

Подставив последние два неравенства в оценку (21), получим требуемое соотношение. Лемма доказана.

Продолжим оценку $\left(19^{\prime}\right)$ величины $p_{1}(\zeta)$. В силу п. 3$)$ леммы 5 получим

$$
p_{1}(\zeta) \leqslant \sum_{0}^{\infty} \frac{d}{2^{n}} \frac{4 d}{2^{n}}+\sum_{-1}^{-\infty} \frac{d}{2^{|n|-1}} \frac{4 d}{2^{|n|-1}}=\frac{32}{3} d^{2}<11 d^{2} .
$$

Пусть $I^{\prime}=\left\{\frac{\pi}{2}-\theta, \theta \in I\right\}$, где интервал $I \subset\left(\varphi_{-}, \varphi_{+}\right)$определен в теореме 3 . Тогда $I^{\prime} \subset\left(\theta_{-}, \theta_{+}\right)$и

$$
\begin{aligned}
\int_{I} \mid h(\theta) & \sin \theta \mid \rho\left(g, \operatorname{ctg} \theta, \frac{|h(\theta)|}{|\sin \theta|}\right) d g^{\prime}(\operatorname{ctg} \theta) \\
= & \int_{I^{\prime}}\left|h\left(\frac{\pi}{2}-\varphi\right) \cos \varphi\right| \rho\left(g, \operatorname{tg} \varphi, \frac{\left|h\left(\frac{\pi}{2}-\varphi\right)\right|}{|\cos \varphi|}\right) d g^{\prime}(\operatorname{tg} \varphi) \\
\leqslant & \int_{\theta_{-}}^{\theta_{+}}\left|h\left(\frac{\pi}{2}-\varphi\right) \cos \varphi\right| \rho\left(g, \operatorname{tg} \varphi, \frac{\left|h\left(\frac{\pi}{2}-\varphi\right)\right|}{|\cos \varphi|}\right) d g^{\prime}(\operatorname{tg} \varphi) \leqslant 11 d^{2} .
\end{aligned}
$$


Подставив последние две оценки в соотношения теоремы 3 , получим верхние оценки для функции $p(\zeta)$ :

$$
p(\zeta) \leqslant\left\{\begin{array}{lr}
\frac{528 \operatorname{diam}^{4}(D)}{|D|^{2}} d^{2}(\zeta), & \text { если } \operatorname{dist}(\zeta) \leqslant \frac{\sigma(D)}{2}, \\
\frac{4 \operatorname{diam}^{2}(D) l(D)}{\sigma^{2}(D)|D|} d^{3}(\zeta)+\frac{528 \operatorname{diam}^{4}(D)}{|D|^{2}} d^{2}(\zeta), & \text { если } \operatorname{dist}(\zeta)>\frac{\sigma(D)}{2} .
\end{array}\right.
$$

Далее на основе соотношения $\left(19^{\prime \prime}\right)$ получим нижние оценки функции $p_{1}(\zeta)$ и, соответственно, функции $p(\zeta)$. Сначала докажем еще одно геометрическое утверждение.

УТВЕРЖДЕНИЕ 3. Если $d(\zeta) \leqslant \sigma(D) / 2$, то имеет место неравенство

$$
\min \left(-\operatorname{tg} \varphi_{-1}, \operatorname{tg} \varphi_{1}\right) \leqslant \frac{5 \operatorname{diam}(D)}{2 \sigma(D)} .
$$

ДокАЗАТЕЛЬСтво. Вернемся к системе координат, связанной с точкой $\zeta$. Проведем опорные прямые $L\left(\frac{\pi}{2}-\varphi_{1}\right)$ и $L\left(\frac{\pi}{2}-\varphi_{-1}\right)$. По определению эти опорные прямые образуют с осью абсцисс углы, равные соответственно $\varphi_{1}$ и $\varphi_{-1}$, причем $-\frac{\pi}{2} \leqslant \varphi_{-1}<0<\varphi_{1} \leqslant \frac{\pi}{2}$. Кроме того, $g\left(\operatorname{tg} \varphi_{ \pm 1}\right)=\frac{d}{2}$. В частности, прямые $L\left(\frac{\pi}{2}-\varphi_{1}\right)$ и $L\left(\frac{\pi}{2}-\varphi_{-1}\right)$ пересекают ось ординат в одной и той же точке $\left(0 ; \frac{d}{2}\right)$. Обозначим эту точку через $A$. Проведем опорную прямую $L\left(-\frac{\pi}{2}\right)$. Это - горизонтальная касательная к области $D$, направленная влево. Пересечение опорных прямых $L\left(\frac{\pi}{2}-\varphi_{-1}\right)$ и $L\left(-\frac{\pi}{2}\right)$ обозначим через $B$, пересечение прямых $L\left(\frac{\pi}{2}-\varphi_{1}\right)$ и $L\left(-\frac{\pi}{2}\right)$ - через $C$, пересечение прямой $B C$ с осью ординат обозначим через $D$ и, наконец, точку опоры прямой $L\left(\frac{\pi}{2}\right)$ (она лежит на оси ординат) обозначим через $O$. Тогда имеем очевидные неравенства

$$
\begin{gathered}
|B C|=|B D|+|D C| \geqslant \sigma(D), \\
|A D|=|A O|+|O D|=\frac{d}{2}+R_{-\frac{\pi}{2}} \leqslant \frac{\sigma(D)}{4}+\operatorname{diam}(D) \leqslant \frac{5}{4} \operatorname{diam}(D) .
\end{gathered}
$$

Поэтому

$$
\operatorname{tg}\left(\frac{\pi}{2}-\varphi_{1}\right)=\frac{|D C|}{|A D|} \geqslant \frac{4|D C|}{5 \operatorname{diam}(D)}, \quad \operatorname{tg}\left(\frac{\pi}{2}+\varphi_{-1}\right)=\frac{|D B|}{|A D|} \geqslant \frac{4|D B|}{5 \operatorname{diam}(D)} .
$$

Суммируя эти оценки, получим

$$
\begin{aligned}
2 \max & \left(\operatorname{tg}\left(\frac{\pi}{2}-\varphi_{1}\right), \operatorname{tg}\left(\frac{\pi}{2}+\varphi_{-1}\right)\right) \\
& \geqslant \operatorname{tg}\left(\frac{\pi}{2}-\varphi_{1}\right)+\operatorname{tg}\left(\frac{\pi}{2}+\varphi_{-1}\right) \geqslant \frac{4|B C|}{5 \operatorname{diam}(D)} \geqslant \frac{4 \sigma(D)}{5 \operatorname{diam}(D)} .
\end{aligned}
$$

Отсюда следует требуемое неравенство. Утверждение доказано.

Далее, не уменьшая общности, будем считать, что

$$
\min \left(-\operatorname{tg} \varphi_{-1}, \operatorname{tg} \varphi_{1}\right)=\operatorname{tg} \varphi_{1}
$$


Для сокращения записи введем обозначение $\rho=\rho\left(g, 0, \frac{d}{2}\right)$. По определению величины $\rho\left(g, 0, \frac{d}{2}\right)$ имеем

$$
g(\rho)+g(-\rho)-2 g(0)=\frac{d}{2} .
$$

С другой стороны,

$$
g(0)=\max _{X_{1} \leqslant x \leqslant X_{2}}(-f(x))=-\min _{X_{1} \leqslant x \leqslant X_{2}} f(x)=-f(0)=-d .
$$

Поэтому должно выполняться равенство

$$
g(\rho)+g(-\rho)=-\frac{3}{2} d
$$

По определению углов $\varphi_{ \pm 1}$ имеем

$$
g\left(\operatorname{tg} \varphi_{1}\right)+g\left(-\operatorname{tg} \varphi_{1}\right)=-\frac{d}{2}+g\left(-\operatorname{tg} \varphi_{1}\right) \geqslant-\frac{d}{2}+g(0)=-\frac{3}{2} d .
$$

Следовательно,

$$
\operatorname{tg} \varphi_{1} \geqslant \rho=\rho\left(g, 0, \frac{d}{2}\right) .
$$

Отсюда и из неравенства $\left(19^{\prime \prime}\right)$ получим

$$
p_{1}(\zeta) \geqslant \frac{d}{2} \int_{-\operatorname{tg} \varphi_{1}}^{\operatorname{tg} \varphi_{1}} \frac{1}{1+t^{2}} \rho\left(g, t, \frac{d}{2}\right) d g^{\prime}(t) \geqslant \frac{d}{2\left(1+\operatorname{tg}^{2} \varphi_{1}\right)} \int_{-\rho}^{\rho} \rho\left(g, t, \frac{d}{2}\right) d g^{\prime}(t) .
$$

Теперь можно воспользоваться утверждением 2 для оценки сверху $\operatorname{tg} \varphi_{1}$ и леммой 5 для оценки снизу интеграла:

$$
p_{1}(\zeta) \geqslant \frac{d}{2} \frac{4 \sigma^{2}(D)}{25 \operatorname{diam}^{2}(D)} \frac{d}{2}=\frac{\sigma^{2}(D)}{25 \operatorname{diam}^{2}(D)} d^{2} .
$$

Эта оценка вместе с соотношением (23) позволяет вывести из теоремы 3 следуюшую теорему.

Теорема 4. Для точек $\zeta$ таких, что $d(\zeta) \leqslant \sigma(D) / 2$, выполнены неравенства

$$
\frac{\sigma^{2}(D)}{113 \operatorname{diam}^{2}(D)} d^{2}(\zeta) \leqslant p(\zeta) \leqslant \frac{528 \operatorname{diam}^{4}(D)}{|D|^{2}} d^{2}(\zeta)
$$

Для точек $\zeta$ таких, что $d(\zeta) \geqslant \sigma(D) / 2$, имеет место оченка сверху

$$
p(\zeta) \leqslant \frac{4 \operatorname{diam}^{2}(D) l(D)}{\sigma^{2}(D)|D|} d^{3}(\zeta)+\frac{528 \operatorname{diam}^{4}(D)}{|D|^{2}} d^{2}(\zeta) .
$$

Здесь $\sigma(D)$ - наименьшая ширина области $D$ по направлениям, $d(\zeta)$ - расстояние от точки $\zeta$ до области $D,|D|-$ площадь $D, l(D)-$ длина границы $D$ $u$, наконеи, $\operatorname{diam}(D)-$ диаметр $D$.

В теореме $1^{\prime}$ мы доказали эквивалентность нормы целой функции $F$ и интегральной нормы для ассоциированной по Борелю функции $\gamma$ с весовой функцией $p(\zeta)$. Все дальнейшее исследование привело к теореме 4 . Оценки снизу 
функции $p(\zeta)$ через $\operatorname{dist}^{2}(\zeta)$ получены только в окрестности области $D$, а оценки сверху - со слагаемым $\operatorname{dist}^{3}(\zeta)$. Следующая теорема позволяет локализовать интегралы по $\mathbb{C} \backslash \bar{D}$ на интегралы по множеству $\Omega \backslash \bar{D}$, где $\Omega$ - произвольная окрестность области $\bar{D}$.

Через $B(z, r)$ обозначим круг с центром в точке $z$ радиуса $r$; если $z=0$, то эту точку не будем указывать.

Пусть

$$
R(D)=\inf \{R>0: D \subset \overline{B(R)}\} .
$$

Теорема 5. Для любого $\varepsilon>0$ через $D_{\varepsilon}$ обозначим область

$$
D_{\varepsilon}=\{\zeta \in \mathbb{C} \backslash \bar{D}: \operatorname{dist}(\zeta, D)<\varepsilon\} \cup D
$$

Тогда если $\gamma \in H_{0}(\mathbb{C} \backslash \bar{D}), \varepsilon \in(0,3 R(D)) u$

$$
\int_{\mathbb{C} \backslash \bar{D}}\left|\gamma^{\prime \prime}(\zeta)\right|^{2} d^{2}(\zeta) d v(\zeta)<\infty
$$

mo

$$
\begin{aligned}
& \int_{\mathbb{C} \backslash \bar{D}}\left|\gamma^{\prime \prime}(\zeta)\right|^{2} d^{2}(\zeta) d v(\zeta) \\
& \quad \leqslant 13\left(\frac{16^{3}(l(D)+\pi \varepsilon)^{2} R^{2}(D)}{\pi^{2} \varepsilon^{4}}+1\right) \int_{D_{\varepsilon} \backslash \bar{D}}\left|\gamma^{\prime \prime}(\zeta)\right|^{2} d^{2}(\zeta) d v(\zeta)
\end{aligned}
$$

$u$

$$
\begin{aligned}
& \int_{\mathbb{C} \backslash \bar{D}}\left|\gamma^{\prime \prime}(\zeta)\right|^{2} d^{3}(\zeta) d v(\zeta) \\
& \quad \leqslant 96 R(D)\left(\frac{5 \cdot 16^{3}(l(D)+\pi \varepsilon)^{2} R^{3}(D)}{\pi^{2} \varepsilon^{4}}+1\right) \int_{D_{\varepsilon} \backslash \bar{D}}\left|\gamma^{\prime \prime}(\zeta)\right|^{2} d^{2}(\zeta) d v(\zeta) .
\end{aligned}
$$

ДокАЗАТЕЛЬСтво. Доказательство проведем в два этапа: на первом этапе (лемма 6) интеграл от $\left|\gamma^{\prime \prime}(\zeta)\right|$ по внешности круга $\overline{B(4 R(D))}$ оценим сверху интегралом по множеству $B(4 R(D)) \backslash \bar{D}$. Затем (лемма 7) интеграл по $B(4 R(D)) \backslash \bar{D}$ оценим сверху интегралом по $D_{\varepsilon} \backslash \bar{D}$.

Далее число $R(D)$ будем обозначать через $R$.

Лемма 6. Имеют место соотношения

$$
\begin{aligned}
& \int_{|\zeta| \geqslant 4 R}\left|\gamma^{\prime \prime}(\zeta)\right|^{2} d^{2}(\zeta) d v(\zeta) \leqslant 12 \int_{B(4 R) \backslash \bar{D}}\left|\gamma^{\prime \prime}(\zeta)\right|^{2} d^{2}(\zeta) d v(\zeta), \\
& \int_{|\zeta| \geqslant 4 R}\left|\gamma^{\prime \prime}(\zeta)\right|^{2} d^{3}(\zeta) d v(\zeta) \leqslant 96 R \int_{B(4 R) \backslash \bar{D}}\left|\gamma^{\prime \prime}(\zeta)\right|^{2} d^{2}(\zeta) d v(\zeta)
\end{aligned}
$$


ДокАЗАТЕЛЬСТво. Представим функцию $\gamma(\zeta)$ в виде ряда Лорана:

$$
\gamma(\zeta)=\sum_{k=0}^{\infty} \frac{\gamma_{k}}{\zeta^{k+1}}, \quad|\zeta|>R
$$

По условиям леммы этот ряд, как и ряд

$$
\gamma^{\prime \prime}(\zeta)=\sum_{k=0}^{\infty} \frac{(k+2)(k+1) \gamma_{k}}{\zeta^{k+3}}=\sum_{k=0}^{\infty} \frac{\gamma_{k}^{\prime \prime}}{\zeta^{k+3}}, \quad|\zeta|>R,
$$

сходится равномерно на множестве $\mathbb{C} \backslash B(2 R)$. Для $\zeta,|\zeta| \geqslant 4 R$, имеем $d(\zeta) \leqslant$ $|\zeta|+R \leqslant \frac{5}{4}|\zeta|$, поэтому

$$
\int_{|\zeta| \geqslant 4 R}\left|\gamma^{\prime \prime}(\zeta)\right|^{2} d^{2}(\zeta) d v(\zeta) \leqslant 2 \int_{|\zeta| \geqslant 4 R}\left|\gamma^{\prime \prime}(\zeta)\right|^{2}|\zeta|^{2} d v(\zeta) .
$$

Переходя в последнем интеграле к полярным координатам и учитывая ортогональность системы $e^{i k \varphi}$ относительно меры $d \varphi$ по $[0,2 \pi]$, получим

$$
\int_{|\zeta| \geqslant 4 R}\left|\gamma^{\prime \prime}(\zeta)\right|^{2} d^{2}(\zeta) d v(\zeta) \leqslant 4 \pi \int_{4 R}^{\infty} \sum_{k=0}^{\infty} \frac{\left|\gamma_{k}^{\prime \prime}\right|^{2} r^{3} d r}{r^{2(k+3)}}=4 \pi \sum_{k=0}^{\infty} \frac{\left|\gamma_{k}^{\prime \prime}\right|^{2}}{2(k+1)(4 R)^{2 k+2}}
$$

Аналогично получается оценка

$$
\int_{|\zeta| \geqslant 4 R}\left|\gamma^{\prime \prime}(\zeta)\right|^{2} d^{3}(\zeta) d v(\zeta) \leqslant 4 \pi \sum_{k=0}^{\infty} \frac{\left|\gamma_{k}^{\prime \prime}\right|^{2}}{(2 k+1)(4 R)^{2 k+1}}
$$

Поскольку при $k \geqslant 0$, очевидно, $\frac{2 k+2}{2 k+1} \leqslant 2$, то

$$
\int_{|\zeta| \geqslant 4 R}\left|\gamma^{\prime \prime}(\zeta)\right|^{2} d^{3}(\zeta) d v(\zeta) \leqslant 32 \pi R \sum_{k=0}^{\infty} \frac{\left|\gamma_{k}^{\prime \prime}\right|^{2}}{2(k+1)(4 R)^{2 k+2}}
$$

Снова с помошью ряда Лорана оценим снизу интеграл по кольцу $B(4 R) \backslash B(2 R)$. При этом учтем, что для $\zeta,|\zeta| \in[2 R, 4 R)$, выполнена оценка $d(\zeta) \geqslant R \geqslant|\zeta| / 4$ :

$$
\begin{aligned}
\int_{2 R \leqslant|\zeta|<4 R}\left|\gamma^{\prime \prime}(\zeta)\right|^{2} d^{2}(\zeta) d v(\zeta) \geqslant \frac{1}{16} & \int_{2 R \leqslant|\zeta|<4 R}\left|\gamma^{\prime \prime}(\zeta)\right|^{2}|\zeta|^{2} d v(\zeta) \\
= & \frac{\pi}{8} \sum_{k=0}^{\infty}\left|\gamma_{k}^{\prime \prime}\right|^{2} \int_{2 R}^{4 R} \frac{d r}{r^{2 k+3}}=\frac{\pi}{8} \sum_{k=0}^{\infty} \frac{\left|\gamma_{k}^{\prime \prime}\right|^{2}}{2(k+1)(4 R)^{2 k+2}}\left(2^{2 k+2}-1\right) \\
& \geqslant \frac{\pi}{3} \sum_{k=0}^{\infty} \frac{\left|\gamma_{k}^{\prime \prime}\right|^{2}}{2(k+1)(4 R)^{2 k+2}} .
\end{aligned}
$$

Отсюда и из неравенства (23) вытекает оценка

$$
\begin{aligned}
\int_{|\zeta| \geqslant 4 R}\left|\gamma^{\prime \prime}(\zeta)\right|^{2} d^{2}(\zeta) d v(\zeta) & \leqslant 12 \int_{2 R \leqslant|\zeta|<4 R}\left|\gamma^{\prime \prime}(\zeta)\right|^{2} d^{2}(\zeta) d v(\zeta) \\
& \leqslant 12 \int_{B(4 R) \backslash \bar{D}}\left|\gamma^{\prime \prime}(\zeta)\right|^{2} d^{2}(\zeta) d v(\zeta),
\end{aligned}
$$


а из неравенства (24) получим

$$
\begin{aligned}
\int_{|\zeta| \geqslant 4 R}\left|\gamma^{\prime \prime}(\zeta)\right|^{2} d^{3}(\zeta) d v(\zeta) & \leqslant 96 R \int_{2 R \leqslant|\zeta|<4 R}\left|\gamma^{\prime \prime}(\zeta)\right|^{2} d^{2}(\zeta) d v(\zeta) \\
& \leqslant 96 R \int_{B(4 R) \backslash \bar{D}}\left|\gamma^{\prime \prime}(\zeta)\right|^{2} d^{2}(\zeta) d v(\zeta)
\end{aligned}
$$

Лемма доказана.

ЛЕмма 7. В условиях теоремы 5 имеют место соотношения

$$
\begin{gathered}
\int_{B(4 R) \backslash \bar{D}_{\varepsilon}}\left|\gamma^{\prime \prime}(\zeta)\right|^{2} d^{2}(\zeta) d v(\zeta) \leqslant \frac{16^{3}(l(D)+\pi \varepsilon)^{2} R^{2}}{\pi^{2} \varepsilon^{4}} \int_{D_{\varepsilon} \backslash \bar{D}}\left|\gamma^{\prime \prime}(\zeta)\right|^{2} d^{2}(\zeta) d v(\zeta) \\
\int_{B(4 R) \backslash \bar{D}_{\varepsilon}}\left|\gamma^{\prime \prime}(\zeta)\right|^{2} d^{3}(\zeta) d v(\zeta) \\
\leqslant \frac{5 \cdot 16^{3}(l(D)+\pi \varepsilon)^{2} R^{3}}{\pi^{2} \varepsilon^{4}} \int_{D_{\varepsilon} \backslash \bar{D}}\left|\gamma^{\prime \prime}(\zeta)\right|^{2} d^{2}(\zeta) d v(\zeta)
\end{gathered}
$$

ДоказАТЕЛЬСтво. Легко заметить, что если $A+B=\{z+w: z \in A, w \in B\}$ для двух множеств $A, B$, то $D_{\varepsilon}=D+B(\varepsilon)$ и $D_{\varepsilon_{1}}+D_{\varepsilon_{2}}=D_{\varepsilon_{1}+\varepsilon_{2}}$ для любых $\varepsilon_{1}, \varepsilon_{2}>0$.

Кроме того,

$$
l\left(D_{\varepsilon}\right)=l(D)+2 \pi \varepsilon .
$$

Если $\zeta \notin \bar{D}_{\varepsilon}$, при этом $\operatorname{dist}\left(\zeta, D_{\varepsilon}\right)=|\zeta-z|$, где $z \in \partial D_{\varepsilon}$, и $\operatorname{dist}(z, D)=\left|z-z_{0}\right|$, где $z_{0} \in \partial D$, то

$$
\operatorname{dist}(\zeta, D) \leqslant\left|\zeta-z_{0}\right| \leqslant|\zeta-z|+\left|z-z_{0}\right| \leqslant \operatorname{dist}\left(\zeta, D_{\varepsilon}\right)+\varepsilon .
$$

Пусть $\zeta \notin \bar{D}_{\varepsilon}$. По формуле Коши имеем оценку сверху:

$$
\begin{aligned}
\left|\gamma^{\prime \prime}(\zeta)\right| & \leqslant \frac{1}{2 \pi}\left|\int_{\partial D_{\varepsilon / 2}} \frac{\gamma^{\prime \prime}(z) d z}{z-\zeta}\right| \leqslant \frac{l\left(D_{\varepsilon / 2}\right)}{2 \pi \operatorname{dist}\left(\zeta, D_{\varepsilon / 2}\right)} \max _{z \in \partial D_{\varepsilon / 2}}\left|\gamma^{\prime \prime}(z)\right| \\
& \leqslant \frac{l(D)+\pi \varepsilon}{2 \pi(\operatorname{dist}(\zeta, D)-\varepsilon / 2)} \max _{z \in \partial D_{\varepsilon / 2}}\left|\gamma^{\prime \prime}(z)\right| .
\end{aligned}
$$

Очевидно, что для $\zeta \notin \bar{D}_{\varepsilon}$ имеем $\operatorname{dist}(\zeta, D) \geqslant \varepsilon$ и при $x \geqslant \varepsilon$

$$
\frac{x}{x-\varepsilon / 2} \leqslant 2 \text {. }
$$

Поэтому

$$
\left|\gamma^{\prime \prime}(\zeta)\right|^{2} d^{2}(\zeta) \leqslant \frac{(l(D)+\pi \varepsilon)^{2}}{\pi^{2}} \max _{z \in \partial D_{\varepsilon / 2}}\left|\gamma^{\prime \prime}(z)\right|^{2}, \quad \zeta \notin \bar{D}_{\varepsilon} .
$$


Если $z \in \partial D_{\varepsilon / 2}$, то круг $z+B(\varepsilon / 4)$ лежит в области $D_{3 \varepsilon / 4} \backslash \bar{D}$. Пользуясь субгармоничностью функции $\left|\gamma^{\prime \prime}(z)\right|^{2}$, получим оценку сверху:

$$
\begin{aligned}
\left|\gamma^{\prime \prime}(z)\right|^{2} & \leqslant \frac{16}{\pi \varepsilon^{2}} \int_{z+B(\varepsilon / 4)}\left|\gamma^{\prime \prime}(w)\right|^{2} d v(w) \\
& \left.\leqslant \frac{16}{\pi \varepsilon^{2}} \int_{w \in z+B(\varepsilon / 4)} d^{-2}(w)\right) \int_{z+B(\varepsilon / 4)}\left|\gamma^{\prime \prime}(w)\right|^{2} d^{2}(w) d v(w) \\
& \leqslant \frac{256}{\pi \varepsilon^{4}} \int_{D_{\varepsilon} \backslash \bar{D}}\left|\gamma^{\prime \prime}(w)\right|^{2} d^{2}(w) d v(w) .
\end{aligned}
$$

Отсюда

$$
\max _{z \in \partial D_{\varepsilon / 2}}\left|\gamma^{\prime \prime}(z)\right|^{2} \leqslant \frac{256}{\pi \varepsilon^{4}} \int_{D_{\varepsilon} \backslash \bar{D}}\left|\gamma^{\prime \prime}(w)\right|^{2} d^{2}(w) d v(w) .
$$

Подставив эту оценку в $(26)$ и интегрируя по $B(4 R) \backslash \bar{D}_{\varepsilon}$, получим первое неравенство леммы. Второе неравенство леммы следует из первого, если заметить, что для $\zeta \in B(4 R) \backslash \bar{D}$ имеем $d(\zeta) \leqslant 5 R$, поэтому

$$
\int_{B(4 R) \backslash \bar{D}_{\varepsilon}}\left|\gamma^{\prime \prime}(\zeta)\right|^{2} d^{3}(\zeta) d v(\zeta) \leqslant 5 R \int_{B(4 R) \backslash \bar{D}_{\varepsilon}}\left|\gamma^{\prime \prime}(\zeta)\right|^{2} d^{2}(\zeta) d v(\zeta) .
$$

Лемма доказана.

Теперь на основе лемм 6 и 7 закончим доказательство теоремы 5 .

Введем множества $G_{1}=\mathbb{C} \backslash B(4 R), G_{2}=B(4 R) \backslash D_{\varepsilon}, G_{3}=D_{\varepsilon} \backslash \bar{D}$. Заметим, что в силу условия $\varepsilon<3 R$ область $D_{\varepsilon}$ лежит в круге $B(4 R)$. Поэтому

$$
\begin{aligned}
\int_{\mathbb{C} \backslash \bar{D}}\left|\gamma^{\prime \prime}(\zeta)\right|^{2} d^{2}(\zeta) d v(\zeta)= & \int_{G_{1}}\left|\gamma^{\prime \prime}(\zeta)\right|^{2} d^{2}(\zeta) d v(\zeta)+\int_{G_{2}}\left|\gamma^{\prime \prime}(\zeta)\right|^{2} d^{2}(\zeta) d v(\zeta) \\
& +\int_{G_{3}}\left|\gamma^{\prime \prime}(\zeta)\right|^{2} d^{2}(\zeta) d v(\zeta) .
\end{aligned}
$$

По лемме 6 оценим первый интеграл справа. Получим

$$
\int_{\mathbb{C} \backslash \bar{D}}\left|\gamma^{\prime \prime}(\zeta)\right|^{2} d^{2}(\zeta) d v(\zeta) \leqslant 13 \int_{G_{2}}\left|\gamma^{\prime \prime}(\zeta)\right|^{2} d^{2}(\zeta) d v(\zeta)+13 \int_{G_{3}}\left|\gamma^{\prime \prime}(\zeta)\right|^{2} d^{2}(\zeta) d v(\zeta)
$$

Далее, на основе первого неравенства леммы 7 оценим интеграл по $G_{2}$ :

$$
\begin{aligned}
\int_{\mathbb{C} \backslash \bar{D}}\left|\gamma^{\prime \prime}(\zeta)\right|^{2} d^{2}(\zeta) d v(\zeta) & \\
& \leqslant 13\left(\frac{16^{3}(l(D)+\pi \varepsilon)^{2} R^{2}}{\pi^{2} \varepsilon^{4}}+1\right) \int_{D_{\varepsilon}}\left|\gamma^{\prime \prime}(\zeta)\right|^{2} d^{2}(\zeta) d v(\zeta) .
\end{aligned}
$$

Точно так же получим второе неравенство теоремы 5 .

Теперь на основе теорем $1^{\prime}, 4,5$ мы можем доказать подготовительную теорему. Итак, пусть $F$ - целая функция, удовлетворяющая условию

$$
\|F\|^{2}=\int_{0}^{2 \pi} \int_{0}^{\infty} \frac{\left|F\left(r e^{i \varphi}\right)\right|^{2}}{K\left(r e^{i \varphi}\right)} d r d \Delta(\varphi)<\infty
$$


a $\gamma$ - функция, ассоциированная по Борелю с $F$. По теореме $1^{\prime}$ имеем

$$
\begin{aligned}
\|F\|^{2} & \geqslant \frac{1}{9 A} \int_{\mathbb{C} \backslash \bar{D}}\left|\gamma^{\prime \prime}(\zeta)\right|^{2} p(\zeta) d v(\zeta), \\
\|F\|^{2} & \leqslant \frac{88}{a} \int_{\mathbb{C} \backslash \bar{D}}\left|\gamma^{\prime \prime}(\zeta)\right|^{2} p(\zeta) d v(\zeta) .
\end{aligned}
$$

Для сокрашения записи введем обозначение $\varepsilon=\sigma(D) / 2$. По теореме 4 на множестве $D_{\varepsilon} \backslash \bar{D}$ имеем оценку снизу для функции $p(\zeta)$ :

$$
p(\zeta) \geqslant \frac{\sigma^{2}(D)}{113 \operatorname{diam}^{2}(D)} d^{2}(\zeta)
$$

которая вместе с неравенством $\left(27^{\prime}\right)$ влечет оценку снизу для $\|F\|$ :

$$
\|F\|^{2} \geqslant \frac{\sigma^{2}(D)}{1017 A \operatorname{diam}^{2}(D)} \int_{D_{\varepsilon} \backslash \bar{D}}\left|\gamma^{\prime \prime}(\zeta)\right|^{2} d^{2}(\zeta) d v(\zeta) .
$$

Наконец, отсюда и из первого неравенства теоремы 5 получим

$$
\|F\|^{2} \geqslant \frac{2^{-14} \sigma^{2}(D)}{A \operatorname{diam}^{2}(D)}\left(\frac{2^{12}(l(D)+\pi \varepsilon)^{2} R^{2}(D)}{\pi^{2} \varepsilon^{4}}+1\right)^{-1} \int_{\mathbb{C} \backslash \bar{D}}\left|\gamma^{\prime \prime}(\zeta)\right|^{2} d^{2}(\zeta) d v(\zeta) .
$$

Учитывая, что $\varepsilon=\sigma(D) / 2$, получим нижнюю оценку в подготовительной теореме.

Из $\left(27^{\prime \prime}\right)$ и оценки сверху функции $p(\zeta)$ в теореме 4 следует оценка сверху для $\|F\|$ :

$$
\begin{aligned}
\|F\|^{2} \leqslant & \frac{352 \operatorname{diam}^{2}(D) l(D)}{a \sigma^{2}(D)|D|} \int_{\mathbb{C} \backslash \bar{D}}\left|\gamma^{\prime \prime}(\zeta)\right|^{2} d^{3}(\zeta) d v(\zeta) \\
& +\frac{2^{17} \operatorname{diam}^{4}(D)}{a|D|^{2}} \int_{\mathbb{C} \backslash \bar{D}}\left|\gamma^{\prime \prime}(\zeta)\right|^{2} d^{2}(\zeta) d v(\zeta) .
\end{aligned}
$$

В силу второго неравенства теоремы 5 с $\varepsilon=\sigma(D) / 2$ получим требуемую верхнюю оценку в подготовительной теореме. Доказательство подготовительной теоремы завершено.

Как уже говорилось во введении, для вывода основной теоремы из подготовительной кроме теоремы А необходимо еще доказать эквивалентность нормы

$$
\|\gamma\|=\left(\int_{\mathbb{C} \backslash \bar{D}}\left|\gamma^{\prime \prime}(\zeta)\right|^{2} d^{2}(\zeta) d v(\zeta)\right)^{1 / 2}
$$

и нормы пространства $B_{2}^{1}(\mathbb{C} \backslash \bar{D})$.

Теорема 6. Существует абсолютная постоянная $c>0$ такая, ито для любой функиии $\gamma \in B_{2}^{1}(\mathbb{C} \backslash \bar{D})$ выполнено соотношение

$$
c \int_{\mathbb{C} \backslash \bar{D}}\left|\gamma^{\prime \prime}(\zeta)\right|^{2} d^{2}(\zeta) d v(\zeta) \leqslant \int_{\mathbb{C} \backslash \bar{D}}\left|\gamma^{\prime}(\zeta)\right|^{2} d v(\zeta) \leqslant 2 \int_{\mathbb{C} \backslash \bar{D}}\left|\gamma^{\prime \prime}(\zeta)\right|^{2} d^{2}(\zeta) d v(\zeta)
$$


ДоКАЗАТЕЛЬСТВо. Воспользуемся теоремой продолжения типа Уитни (см. [16, с. 203]).

Teорема С. Пусть $F$ - произвольное замкнутое множсество в $\mathbb{R}^{n}$. Тогда на $\mathbb{R}^{n} \backslash F$ существует бесконечно дифференцируемая функиия $\delta(x)=\delta(x, F)$, обладающая свойствами

$$
c_{1} \delta(x) \leqslant \operatorname{dist}(x, F) \leqslant c_{2} \delta(x)
$$

и для любого а имеем

$$
\left|\frac{\partial^{\alpha}}{\partial x^{\alpha}} \delta(x)\right| \leqslant B_{\alpha}(\operatorname{dist}(x, F))^{1-|\alpha|},
$$

где $B_{\alpha}, c_{1}, c_{2}$ не зависят от $F$.

Возьмем выпуклый многоугольник $D^{\prime}$, содержащий $\bar{D}$ и содержащийся в $B(2 R(D))$. Пусть $\delta(z)$ - гладкая функция, эквивалентная $\operatorname{dist}\left(z, \bar{D}^{\prime}\right)$, существование которой обеспечивается теоремой С. Заметим, что имеет место равенство

$$
\left|\gamma^{\prime \prime}\right|^{2}=\frac{1}{4} \Delta\left|\gamma^{\prime}\right|^{2}
$$

где $\Delta=\frac{\partial^{2}}{\partial x^{2}}+\frac{\partial^{2}}{\partial y^{2}}-$ оператор Лапласа. Возьмем число $R>2 R(D)$. Граница области $G=\left(\mathbb{C} \backslash \bar{D}^{\prime}\right) \cap B(R)$ кусочно гладкая, поэтому в этой области к функциям $\left|\gamma^{\prime}(z)\right|^{2}$ и $\delta^{2}(z)$ можно применить формулу Грина:

$$
\begin{aligned}
\int_{G}\left(\delta^{2}(z) \Delta\left|\gamma^{\prime}(z)\right|^{2}-\left|\gamma^{\prime}(z)\right|^{2} \Delta \delta^{2}(z)\right) d v(z) \\
=\int_{\partial G}\left(\delta^{2}(z) \frac{\partial\left|\gamma^{\prime}(z)\right|^{2}}{\partial n}-\left|\gamma^{\prime}(z)\right|^{2} \frac{\partial \delta^{2}(z)}{\partial n}\right) d s(z),
\end{aligned}
$$

где $d s(z)$ - элемент длины границы. Интегралы по границе $B(R)$ на основании соотношений

$$
\begin{gathered}
\left|\gamma^{\prime}(z)\right|^{2}=O\left(\frac{1}{R^{4}}\right), \quad\left|\frac{\partial\left|\gamma^{\prime}(z)\right|^{2}}{\partial n}\right|=O\left(\frac{1}{R^{5}}\right), \\
\delta^{2}(z)=O\left(\operatorname{dist}^{2}\left(z, D^{\prime}\right)\right)=O\left(R^{2}\right), \quad\left|\frac{\partial \delta^{2}(z)}{\partial n}\right|=O(R)
\end{gathered}
$$

стремятся к нулю при $R \rightarrow \infty$. На гранище $D^{\prime}$ по свойствам функции $\delta(z)$ имеем

$$
\delta(z) \equiv 0, \quad \frac{\partial \delta^{2}(z)}{\partial n} \equiv 0 .
$$

Поэтому формула Грина выписьвается в виде

$$
4 \int_{\mathbb{C} \backslash \bar{D}^{\prime}}\left|\gamma^{\prime \prime}(z)\right|^{2} \delta^{2}(z) d v(z)=\int_{\mathbb{C} \backslash \bar{D}^{\prime}}\left|\gamma^{\prime}(z)\right|^{2} \Delta \delta^{2}(z) d v(z) .
$$


Теперь снова воспользуемся свойствами функции $\delta^{2}(z)$ :

$$
\delta^{2}(z) \geqslant c_{1}^{2} \operatorname{dist}^{2}\left(z, D^{\prime}\right), \quad\left|\Delta \delta^{2}(z)\right|=\left.|| 2 \operatorname{grad} \delta(z)\right|^{2}+\delta(z) \Delta \delta(z) \mid \leqslant 8 B_{1}^{2}+2 c_{2} B_{2},
$$

где $c_{1}, c_{2}, B_{1}, B_{2}$ - абсолютные постоянные (не зависящие от области $D^{\prime}$ ) из теоремы С. В результате получим

$$
\int_{\mathbb{C} \backslash \bar{D}^{\prime}}\left|\gamma^{\prime \prime}(z)\right|^{2} \operatorname{dist}^{2}\left(z, D^{\prime}\right) d v(z) \leqslant \frac{4 B_{1}^{2}+c_{2} B_{2}}{2 c_{1}^{2}} \int_{\mathbb{C} \backslash \bar{D}^{\prime}}\left|\gamma^{\prime}(z)\right|^{2} d v(z) .
$$

Чтобы получить такую оценку для области $D$, возьмем последовательность вьпуклых многоугольников $D_{n}$ со свойствами

$$
B(2 R(D)) \subset D_{n} \supset \bar{D}, \quad D_{n} \supset \bar{D}_{n+1}, \quad \bigcap_{n=1}^{\infty} D_{n}=\bar{D} .
$$

Для каждого из этих многоугольников выполнена оценка (29). Поскольку постоянная в правой части не зависит от области, то можно перейти к пределу и получить правое неравенство в теореме 6 с постоянной

$$
c=\frac{2 c_{1}^{2}}{4 B_{1}^{2}+c_{2} B_{2}} .
$$

Для доказательства левого неравенства теоремы 6 снова начнем с выпуклого многоугольника $D^{\prime}$, который содержит $\bar{D}$ и содержится в $B(2 R(D))$. Легко проверить, что функция $\operatorname{dist}\left(z, D^{\prime}\right)$ непрерывно дифференцируема в области $\mathbb{C} \backslash \bar{D}^{\prime}$. Для применения формулы Грина этого недостаточно. Поэтому воспользуемся обычным методом сглаживания. Возьмем неотрицательную гладкую функцию $\alpha$ типа "шапочки":

$$
\alpha(z) \equiv 0 \text { при }|z| \geqslant 1, \quad \int_{\mathbb{C}} \alpha(z) d v(z)=1 .
$$

Продолжим функцию $\operatorname{dist}\left(z, D^{\prime}\right)$ нулем на $D^{\prime}$ и для $\varepsilon>0$ рассмотрим функцию

$$
d_{\varepsilon}(z)=\frac{1}{\varepsilon^{2}} \int_{\mathbb{C}} \alpha\left(\frac{z-\zeta}{\varepsilon}\right) \operatorname{dist}\left(\zeta, D^{\prime}\right) d v(\zeta) .
$$

Как известно, функция $d_{\varepsilon}$ оказывается гладкой. Функция $\operatorname{dist}\left(z, D^{\prime}\right)$ - выпуклая, в частности субгармоническая, поэтому функции $d_{\varepsilon}$ тоже оказываются субгармоническими и при $\varepsilon \rightarrow 0$, убывая, сходятся к $\operatorname{dist}\left(z, D^{\prime}\right)$ (см. [17]). Субгармоничность гладкой функции $d_{\varepsilon}$, в частности, означает, что $\Delta d_{\varepsilon}(z) \geqslant 0$. Функция $d_{\varepsilon}(z)$ тождественно равна нулю при $z \in D^{\prime}$ и $\operatorname{dist}\left(z, D^{\prime}\right) \geqslant \varepsilon$. Положим

$$
D^{\varepsilon}=\left\{z \in D^{\prime}: \operatorname{dist}\left(z, D^{\prime}\right)>\varepsilon\right\} .
$$

Очевидно, что область $D^{\varepsilon}$ при малых $\varepsilon>0$ представляет собой выпуклый многоугольник в $D^{\prime}$ со сторонами, параллельными сторонам $D^{\prime}$ и отстояшими от соответствующих сторон на $\varepsilon$. Будем считать, что $\varepsilon<\operatorname{dist}\left(\bar{D}, \partial D^{\prime}\right) ;$ тогда $D^{\varepsilon} \supset \bar{D}$. Так же, как соотношение (28), получим равенство

$$
4 \int_{\mathbb{C} \backslash \bar{D}^{\varepsilon}}\left|\gamma^{\prime \prime}(z)\right|^{2} d_{\varepsilon}^{2}(z) d v(z)=\int_{\mathbb{C} \backslash \bar{D}^{\varepsilon}}\left|\gamma^{\prime}(z)\right|^{2} \Delta d_{\varepsilon}^{2}(z) d v(z) .
$$


В силу субгармоничности функции $d_{\varepsilon}(z)$ имеем

$$
\Delta d_{\varepsilon}^{2}(z)=2\left|\operatorname{grad} d_{\varepsilon}(z)\right|^{2}+d_{\varepsilon}(z) \Delta d_{\varepsilon}(z) \geqslant 2\left|\operatorname{grad} d_{\varepsilon}(z)\right|^{2} .
$$

Следовательно, для любого $\varepsilon>0$ получим оценку

$$
2 \int_{\mathbb{C} \backslash \bar{D}}\left|\gamma^{\prime \prime}(z)\right|^{2} d_{\varepsilon}^{2}(z) d v(z) \geqslant \int_{\mathbb{C} \backslash \bar{D}}\left|\gamma^{\prime}(z)\right|^{2}\left|\operatorname{grad} d_{\mathcal{\varepsilon}}(z)\right|^{2} d v(z) .
$$

Теперь устремим $\varepsilon$ к нулю. В силу непрерьвной дифференцируемости $\operatorname{dist}\left(z, D^{\prime}\right)$ имеем

$$
\frac{\partial d_{\varepsilon}(z)}{\partial x} \rightarrow \frac{\partial \operatorname{dist}\left(z, D^{\prime}\right)}{\partial x}, \quad \frac{\partial d_{\varepsilon}(z)}{\partial y} \rightarrow \frac{\partial \operatorname{dist}\left(z, D^{\prime}\right)}{\partial y} .
$$

Следовательно,

$$
\lim _{\varepsilon \rightarrow 0}\left|\operatorname{grad} d_{\varepsilon}(z)\right|^{2}=\left|\operatorname{grad} \operatorname{dist}\left(z, D^{\prime}\right)\right|^{2} .
$$

Таким образом, в пределе получим оценку

$$
2 \int_{\mathbb{C} \backslash \bar{D}}\left|\gamma^{\prime \prime}(z)\right|^{2} \operatorname{dist}^{2}\left(z, D^{\prime}\right) d v(z) \geqslant \int_{\mathbb{C} \backslash \bar{D}}\left|\gamma^{\prime}(z)\right|^{2}\left|\operatorname{grad} \operatorname{dist}\left(z, D^{\prime}\right)\right|^{2} d v(z) .
$$

Лемма 8. Пусть D-некоторая выпуклая область и $\notin D$. Если функиия $\operatorname{dist}(z, D)$ дифферениируема в точке $\zeta$, то

$$
|\operatorname{grad} \operatorname{dist}(\zeta, D)|=1 \text {. }
$$

ДокАзАТЕЛЬСтво. Как нетрудно убедиться, функция $\operatorname{dist}(z, D)$ липшицева, т. e.

$$
|\operatorname{dist}(z, D)-\operatorname{dist}(w, D)| \leqslant|z-w|,
$$

поэтому любая производная по любому направлению, если она существует, не превосходит 1. С другой стороны, если $z_{0} \in \partial D$ - точка достижения расстояния для $\zeta$, т.е.

$$
\operatorname{dist}(\zeta, D)=\left|\zeta-z_{0}\right|,
$$

то производная по направлению $\left(\zeta-z_{0}\right) /\left|\zeta-z_{0}\right|$ в точке $\zeta$ равна 1 . Поскольку градиент - это наибольшая по модулю производная по направлениям, то

$$
|\operatorname{grad} \operatorname{dist}(\zeta, D)|=1 \text {. }
$$

Из этой леммы и соотношения (30) вытекает оценка

$$
2 \int_{\mathbb{C} \backslash \bar{D}}\left|\gamma^{\prime \prime}(z)\right|^{2} \operatorname{dist}^{2}\left(z, D^{\prime}\right) d v(z) \geqslant \int_{\mathbb{C} \backslash \bar{D}}\left|\gamma^{\prime}(z)\right|^{2} d v(z) .
$$

Остается выбрать последовательность выпуклых многоугольников, стягиваюшихся к области $D$. Теорема 6 доказана. 


\section{Список литературы}

1. Гайер Д. Лекции по теории аппроксимации в комплексной плоскости. М.: Мир, 1986.

2. Луценко В. И., Юлмухаметов Р. С. Теорема Пэли-Винера в пространствах Смирнова // Тр. МИАН. 1991. Т. 200. С. 245-254.

3. Левин Б. Я. Распределение корней целых функций. М.: Гостехиздат, 1956.

4. Лихт М. К. Замечание к теореме Палея и Винера о целых функциях конечной степени // УМН. 1964. Т. 19. № 1. С. 169-171.

5. Кацнельсон В. Э. Обобщение теоремы Винера-Палея о представлении целых функций конечной степени // Теория функций, функц. анализ и их прилож. 1965. №1. С. 99-110.

6. Любарский Ю. И. Теорема Винера-Пэли для выпуклых множеств // Изв. АН АрмССР. 1988. T. 23. № 2. C. 163-172.

7. Напалков B. B., Юлмухаметов P. C. О преобразованиях Гильберта в пространствах Бергмана // Матем. заметки. 2001. Т. 70. № 1. С. 23-30.

8. Меренков C.A. О преобразованиях Коши в пространстве Бергмана // Матем. физика, анализ и геометрия. Харьков. 1999. № 4. С. 23-31.

9. Напалков В.В. (мл.), Юлмухаметов Р. С. О преобразовании Коши функционалов на пространстве Бергмана // Матем. сб. 1994. Т. 185. № 7. С. 77-86.

10. Луценко В.И., Юлмухаметов Р. С. Обобщение теоремы Пэли-Винера на весовые пространства // Матем. заметки. 1990. Т. 48. № 5. С. 139-144.

11. Державец Б. А. Пространства функций, аналитических в выпуклых областях $\mathbb{C}^{n}$ и имеющих заданное поведение вблизи границы // ДАН СССР. 1984. Т. 276. №6. C. $1297-1301$.

12. Напалков В. В. Пространства аналитических функций заданного роста вблизи границы // Изв. АН СССР. Сер. матем. 1987. Т. 51. № 2. С. 287-305.

13. Абузярова Н. Ф., Юлмухаметов Р. С. Сопряженные пространства к весовым пространствам аналитических функций // Сиб. матем. журн. 2001. Т. 42. № 1. С. 3-17.

14. Enuфанов О. В. Двойственность одной пары пространств аналитических функций ограниченного роста // ДАН СССР. 1991. Т. 319. №6. С. 1297-1300.

15. Юлмухаметов Р. С. Пространства аналитических функций, имеющих заданный рост вблизи границы // Матем. заметки. 1982. Т. 32. №1. С. 41-57.

16. Стейн И. Сингулярные интегралы и дифференциальные свойства функций. М.: Мир, 1973.

17. Ронкин Л. И. Введение в теорию целых функций многих переменных. М.: Наука, 1971.

E-mail: Yulmukhametov@bsu.bashedu.ru

Поступило в редакцию

01.04 .2002 